\title{
Endogenous versus exogenous factors: What matters for vent mussel communities?
}

\author{
Sarrazin Jozee ${ }^{1,}{ }^{*}$, Portail Marie ${ }^{1}$, Legrand E. ${ }^{2}$, Cathalot Cecile ${ }^{3}$, Laes Agathe ${ }^{4}$, Lahaye Noe ${ }^{5}$, \\ Sarradin Pierre-Marie ${ }^{1}$, Husson Berengere ${ }^{6}$
}

\author{
${ }^{1}$ Ifremer, Centre de Bretagne, REM/EEP, Laboratoire Environnement Profond, F-29 280, Plouzané, \\ France \\ 2 Institute of Marine Research, Nordnesgaten 50, 5005, Bergen, Norway \\ 3 Ifremer, Centre de Bretagne, REM/GM, Laboratoire Cycles Géochimiques et Ressources, F-29 280, \\ Plouzané, France \\ 4 Ifremer, Centre de Bretagne, REM/RDT, Laboratoire Détection, Capteurs et Mesures, F-29 280, \\ Plouzané, France \\ ${ }^{5}$ Université de Bretagne Occidentale, CNRS, IRD, Ifremer, Laboratoire d'Océanographie Physique et \\ Spatiale (LOPS), IUEM, Brest, France \\ ${ }^{6}$ Institute of Marine Research, Hjalmar Johansens gate 14, 9007, Tromsø, Norway
}

* Corresponding author : Jozee Sarrazin, email address : jozee.sarrazin@ifremer.fr

\begin{abstract}
:
The factors influencing the structure of hydrothermal communities are not necessarily similar across geographical regions and vent fields. In the present study, we explore the role of environmental conditions on vent community structure at local and regional spatial scales, focusing on the assemblages dominated by the engineer species Bathymodiolus azoricus. Their presence in several vent fields on the northern Mid-Atlantic Ridge (MAR) represents a unique opportunity to compare their associated fauna and to better understand large-scale biodiversity patterns. Two types of factors were considered: exogenous ones such as the presence of biogeographical barriers, depth, and distance between sites, and those linked to the intrinsic characteristics of each field (endogenous factors, e.g. local abiotic conditions). The main goals of our study are to describe and compare the biological and environmental characteristics of mussel assemblages collected from three vent fields - Menez Gwen (MG), Lucky Strike (LS) and Rainbow (RB) - located in the Azores Triple Junction on the MAR. These fields differ in the chemistry of their endmember fluids, their depths as well as their geological settings. We focus on the composition, abundance, and diversity of the macro- and meiofauna associated with the vent mussels. Comparing them between vent fields and among the different edifices of a single vent field (Lucky Strike) allows us to study the influence of exogenous and endogenous factors at local and regional scales. On the local scale, our results show that the pool of species associated with $B$. azoricus is largely shared between the edifices of LS, which suggest no obstacles to species dispersion across the field. However, differences in relative abundances foster mussel assemblages with varying diversity, probably linked to differences in local abiotic conditions between the various chemistry domains of the field. At larger scale, we observed a "vent field signature" in the communities associated with B. azoricus. Distinct patterns of abundances and diversity were observed across the three fields, with MG appearing as a particular case. The presence of a longitudinal current to the south of this field and its depth difference may partially explain those
\end{abstract}


discrepancies. This study represents one of the most comprehensive integrated faunal and environmental datasets obtained across hydrothermal vent fields.

\section{Graphical abstract}

The factors influencing the structure of hydrothermal communities are not necessarily similar across geographical regions and vent fields. In the present study, we explore the role of environmental conditions on vent community structure at local and regional spatial scales, focusing on the assemblages dominated by the engineer species Bathymodiolus azoricus. Distinct patterns of faunal abundances and diversity were observed across the three studied vent fields [Menez Gwen (MG), Lucky Strike (LS) and Rainbow (RB)], with MG appearing as a particular case. The presence of a longitudinal current to the south of this field and its depth difference may partially explain those discrepancies.

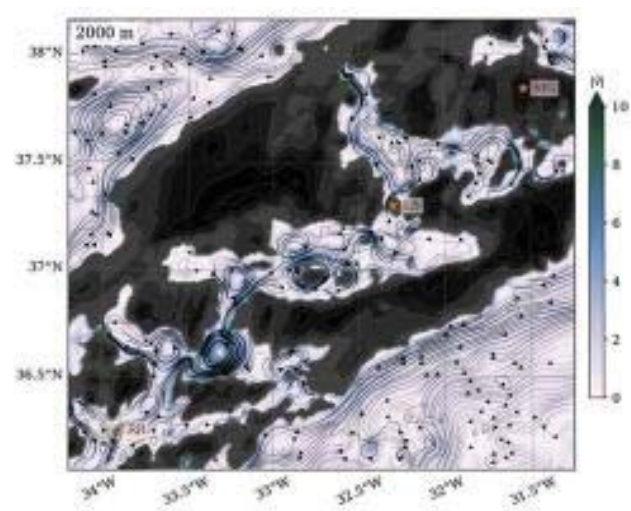

\section{Highlights}

High consistency in species composition across different edifices at the field scale -Lucky Strike. Various chemistry domains may influence local abiotic conditions and diversity patterns. A "vent field signature" is observed across the three studied fields. Distinct abundance and diversity patterns are observed, Menez Gwen being the most different. Exogenous factors better explain the dissimilarities in faunal community structure. Of particular interest are the current patterns between the three vent fields.

Keywords : intra-field variability, inter-field variability, hydrothermal fauna, abiotic factors, diversity measures, macrofauna, meiofauna, environmental drivers, community structure, habitats. 


\section{Introduction}

Compared to the sedimentary environments of the deep ocean, hydrothermal vent ecosystems are spatially limited and host high biomass of endemic fauna (Wright et al., 1993; Van Dover and Trask, 2000). These ecosystems are often dominated by large dominant engineer species (siboglinids, mytilids, alvinocaridids, alvinellids) that live in association with exo- or endosymbiotic microorganisms (Govenar 2012). These foundation species provide a substratum, a refuge, and food to various associated meio- and macrofaunal taxa (Van Dover, 2002, 2003; Zekely et al. 2006; Govenar and Fisher, 2007). They are distributed along a gradient between cold oxygenated seawater $\left(<9^{\circ} \mathrm{C}\right)$ and hot (up to $400^{\circ} \mathrm{C}$ ) acidic hydrothermal fluids (e.g. Gebruk et al., 1997; Marsh et al., 2012; Sen et al., 2013). Over the years, several studies have demonstrated the influence of environmental conditions on species distribution and dynamics at the scale of the large hydrothermal sulfide edifices (e.g. Sarrazin et al., 1997; 1999, Sarrazin and Juniper, 1999 ; Van Dover and Doerries, 2005; Copley et al., 1997, 2007; Cuvelier et al. 2009, 2011a, b, 2014a, b; Fabri et al., 2011; Kim and Hammerstrom, 2012; Sen et al., 2013; Sarrazin et al., 2015) and have shown that edifice 
walls are characterized by a high degree of faunal heterogeneity linked to the patchiness of vent emissions (Sarrazin et al., 1997, Sarrazin and Juniper 1999; Luther et al., 2001; Gollner et al. 2010; Podowski et al., 2010; Fabri et al., 2011; Marsh et al., 2012; Nye et al., 2013; Sarrazin et al., 2015; Husson et al., 2017). Particularly important are the reduced chemical compounds that are involved in chemosynthetic pathways such as hydrogen sulfide, methane, iron and hydrogen (Jannasch 1985, Schmidt et al. 2008, Luther et al. 2012). The concentrations of metals and oxygen are other potential factors influencing species distribution at small-scales (Desbruyères et al. 2000, Martins et al. 2011, Cuvelier et al. 2014b). A few studies have looked at inter-field variations, which can be related to a series of factors including the physical mechanisms involved in their connection or isolation, local abiotic conditions, biological interactions, and habitat instability (see Ramirez-Llodra et al., 2011 for a review). Today, deep-sea mineral resources are increasingly coveted, and it is now urgent to gain a better understanding of the functioning and dynamics of deep-sea faunal communities, particularly at different spatial and temporal scales. However, our knowledge on the role of larger, field-scale patterns on community structure is still limited.

At global scales, it has been shown that the factors influencing the structure of vent communities are not necessarily similar across geographical regions. For example, on the Mid-Atlantic Ridge (MAR), one study demonstrated that the similarity among assemblages of different foundation species within the same hydrothermal field is higher than among assemblages of the same foundation species from different fields and depths, suggesting a strong influence of local field characteristics (Rybakova and Galkin, 2015). On the contrary, on the East Pacific Rise (EPR), similarity is higher among assemblages of the same foundation 
species from different fields located at the same depth than among assemblages of different foundation species from the same field, thus pointing to the role of engineer species in structuring communities (Rybakova and Galkin, 2015). At the ridge scale (MAR), Goroslavskaya and Galkin (2011) showed that faunal diversity between different faunal assemblages was probably influenced by the history of the sites.

In the present study, we continue to explore the role of environmental conditions on vent community structure at larger spatial scales. We considered two types of factors: those linked to exogenous factors such as the presence of biogeographical barriers, depth, and distance between sites, and those linked to the intrinsic characteristics of each field (endogenous factors, e.g. local abiotic conditions). The identification of their respective contributions on community structure is complex due to their close relationships (Portail et al., 2018). Therefore, comparison of faunal assemblages, dominated by the same foundation species across vent fields can be used to discount, at least partially, the factors that may be related to the role of these engineering species and their habitats. Here, we focused on the assemblages dominated by the mytilid Bathymodiolus azoricus. This engineer species forms one of the three faunal assemblages that dominate vent sites of the northern MAR between $23^{\circ} \mathrm{N}$ and $37^{\circ} \mathrm{N}$ (Van Dover, 1995; Desbruyères et al., 2001; Cuvelier et al., 2009; Sarrazin et al., 2015; Husson et al. 2017). The two others are dominated by alvinocaridid shrimp (Desbruyères et al., 2001; Marcon et al., 2013; Sarrazin et al., 2015) and less commonly, by small gastropod assemblages (Sarrazin et al., in preparation). These assemblages colonize active hydrothermal sites patchily and are distributed according to local environmental conditions and distance from fluid emissions (Cuvelier et al., 2009, 2011b). Of these three 
MAR assemblage, those dominated by $B$. azoricus are the most widespread, covering very large surface areas on the active vent sites (Sarrazin, pers. obs.). They offer secondary surfaces for other invertebrates to colonize, contributing to enhanced species diversity (Goroslavskaya and Galkin, 2011). Their presence in several vent fields on the northern MAR represents a unique opportunity to compare their associated fauna and to better understand large-scale biodiversity patterns with respect to environmental conditions.

The main goals of our study are to describe and compare the biological and environmental characteristics of different $B$. azoricus assemblages collected from three vent fields - Menez Gwen (MG), Lucky Strike (LS) and Rainbow (RB) - in the Azores Triple Junction on the MAR (Figure 1A). These vent fields differ in the chemistry of their end-member fluids, their depths as well as their geological settings. Here, we focus on the composition, abundance, and diversity of the macro- and meiofauna associated with the vent mussels. We address the following questions: (1) are there differences in community structure between different edifices in the same vent field?; (2) are there differences in community structure between mussel assemblages from different vent fields?; (3) what are the roles of local (endogenous) and regional (exogenous) environmental conditions in the observed faunal patterns?

\section{Materials and Methods}

\section{Study sites}

The MG vent field $\left(37^{\circ} 50.6^{\prime} \mathrm{N} ; 31^{\circ} 31.5^{\prime} \mathrm{W}\right.$, Figure $\left.1 \mathrm{~B}\right)$ was discovered in 1994 during the DIVA1 cruise. Spreading over $55 \mathrm{~km}$ on the slope of a $700 \mathrm{~m}$ high volcano of the axial valley (Fouquet et al., 1994; Charlou et al., 2000), MG is one of the shallowest known vent system 
of the MAR, with a mean depth of $850 \mathrm{~m}$. The hydrothermally active sites are concentrated on the southern and eastern flanks of this small volcano (Charlou et al., 2000, Desbruyères et al., 2001) and are partly colonized by large assemblages of B. azoricus vent mussels covered by Lepetodrilidae gastropods (Marcon et al., 2013). Shrimp assemblages are visible close to the fluid. A third assemblage, dominated by gastropods and associated with the warmest habitats has been observed on some images (Marcon et al., 2013). The Atos 10 marker site, sampled during this study, is located at a depth of $828 \mathrm{~m}$, and covers an area of $59 \mathrm{~m}^{2}$ (Marcon et al., 2013).

Discovered in 1993 , the $1 \mathrm{~km}^{2}$ wide LS vent field $\left(37^{\circ} 17.5^{\prime} \mathrm{N} ; 32^{\circ} 16.9^{\prime} \mathrm{W}\right.$, Figure $1 \mathrm{C}$ ) lies at 1700 m depth (Langmuir et al. 1997). It is found in the center of three volcanic cones that surround an ancient lava lake. The magmatic chamber is situated at $3 \mathrm{~km}$ depth below the seafloor, feeding about 20 active sulfide edifices (Ondreas et al., 2009). Similar to MG, the faunal assemblages at LS are largely dominated by B. azoricus mussel assemblages and, less commonly, by shrimp and gastropod assemblages (Desbruyères et al., 2001; Cuvelier et al., 2009, 2011a; Sarrazin et al., 2015, Husson et al., 2017, Sarrazin et al., in preparation for gastropods). B. azoricus assemblages at Lucky Strike can be considered a climax-community because their dominance has been observed for several decades (Cuvelier et al., 2011b). Four active edifices of LS were included in the present study: Cypress (CY), Y3, Eiffel Tower (TE) and Montségur (MO).

Discovered in $1997, \mathrm{RB}\left(36^{\circ} 13.8^{\prime} \mathrm{N} ; 33^{\circ}, 54.1^{\prime} \mathrm{W}\right.$, Figure $\left.1 \mathrm{D}\right)$ is the deepest field of this study $(\sim 2300 \mathrm{~m})$. It covers an area of $0.03 \mathrm{~km}^{2}$, harboring approximately 10 active hydrothermal edifices (Marcaillou et al., 2011). Rainbow is one of the five fields of the MAR known to be 
constituted of an ultramafic substratum (Cannat et al., 1997). In terms of fauna, it is colonized by dense assemblages of alvinocaridid shrimp dominated by Rimicaris exoculata as well as by B. azoricus assemblages embedded in sulfide particles (Desbruyères et al., 2001).

A total of 21 B. azoricus faunal assemblages from these three vent fields were examined during the present study (supplementary material Figure S.1.). Their habitats were characterized and the fauna sampled during three different cruises on the MAR: Momarsat 2011, Momarsat 2012, and Biobaz 2013 (Table 1, Figure 1). Biobaz was the only cruise that visited all three vent fields; the two others visited LS only. These cruises took place aboard the French R/Vs Pourquoi pas? (Momarsat 2011 \& Biobaz 2013) and Thalassa (Momarsat 2012) equipped with the remotely operated vehicle (ROV) Victor6000.

\section{Environmental characterization}

Environmental conditions were assessed on all faunal assemblages to identify the structuring factors (temperature variables, depth, geographical distances) and evaluate their effects at the local and regional scales (Table 1). Due to technical constraints, we were not able to gather a complete dataset for chemical conditions as initially planned. To overcome this issue and characterize the environmental conditions within each mussel assemblage, temperature was used as a proxy for hydrothermal influence. Indeed, previous analyses at vents have demonstrated that, at the scale of a site, there is generally a significant correlation between temperature and the concentrations of chemicals (especially hydrogen sulfide, iron and manganese) in vent fluids (Le Bris et al. 2006, Sarrazin et al. 1999, Sarradin et al., 2009, Waeles et al. 2017). 
Autonomous temperature probes (WHOI-MISO low temp-ONSET ${ }^{\circledR}$ ) were deployed within

147

each mussel assemblage at all sites (Table 1). They were directly placed on the mussels when possible or on adjacent mussel assemblages with similar size classes (Cuvelier et al., 2009, Husson et al., 2017). Temperature measurements were taken every $15 \mathrm{~min}$. All probes were collected after one year, except for those on MG, which were collected after 13 days because no future cruise was planned to this field. All temperature measurements were corrected using bottom temperatures as minimum values.

Local (intra-field) scale -At Lucky Strike, temperature measurements were taken every 15 min for all samples, except for $\mathrm{Y} 32$ and $\mathrm{Y} 33$, whose probes recorded temperature every 15 sec. For Y31, the probe was not placed within the small-size class mussels that were sampled, so temperature was obtained with the ROV probe. Temperatures of end-member fluids were measured using the Victor6000 probe for each edifice during routine fluid sampling.

A few $\mathrm{H}_{2} \mathrm{~S}$ measurements were done directly above mussel assemblages and on different edifices using the in situ chemical analyzer CHEMINI (Vuillemin et al. 2009) during the Momarsat cruises (2011 \& 2012). However, due to technical problems, the dataset was incomplete and $\mathrm{H}_{2} \mathrm{~S}$ values could not be used in the statistical analyses. However, they provide some support for the discussion of the results (Table S1).

Regional (inter-field) scale -Most temperature values were obtained with autonomous probes, except those for RB that were obtained during the Momareto cruise (2006) using Victor6000 probe. The habitat chemistry of one mussel assemblage per field was evaluated 
during the Biobaz 2013 cruise using the in situ chemical analyzer CHEMINI (Vuillemin et al., 2009) and the PEPITO water sampler (Sarradin et al., 2009, Table S1). This characterization was done within a single mussel assemblage just before faunal sampling on LS (Eiffel Tower), MG (ATOS 10) and RB (France 5). However, due to technical problems, only methane $\left(\mathrm{CH}_{4}\right)$ and dissolved iron (Fe) concentrations could be evaluated on all fields. For $\mathrm{H}_{2} \mathrm{~S}$ concentrations, only measurements over one assemblage on MG was successful (Table S1). The chemical data included in Table S1, although incomplete, provide some support for the discussion of the results. To complete the picture, we estimated the maximum ranges of $\mathrm{H}_{2} \mathrm{~S}$ available for the fauna based on the average end-member $\mathrm{H}_{2} \mathrm{~S}$ concentration of each vent field extrapolated through simple conservative mixing with background seawater. Temperature (mean \& maximum) was used as the conservative tracer for dilution (Table S1). These estimated concentrations represent "absolute" maximum concentrations available for the fauna and have to be used cautiously. Indeed, as $\mathrm{H}_{2} \mathrm{~S}$ is very reactive, it will be consumed during mixing with seawater through polysulfide precipitation processes. In addition, due to conductive cooling processes in the sub-seafloor, $\mathrm{H}_{2} \mathrm{~S}$ concentrations in the fluids around the fauna may be lower compared to more focused and direct fluid outputs. Finally, the mean environmental conditions of end-member fluids (data from Charlou et al. 2000, 2002 and Douville et al. 2002) and bottom temperatures (measured during the Biobaz cruise (2013) with the Victor6000 probe) for each vent field are reported in Table S1.

\section{Faunal sampling and identification}

Faunal samples were taken from three distinct Bathymodiolus azoricus assemblages on each edifice, for a total of 21 assemblages over three vent fields (see Figure 2 for one 
example/field and Figure S.1. for a photo of each sampling location). Fauna was sampled using the Victor6000 suction sampler and grab arm following the protocol described in Cuvelier et al. (2012). Once brought on board, faunal samples from each location were washed over stacked sieves ( $250 \mu \mathrm{m}$ and $20 \mu \mathrm{m}$ mesh sizes). The fauna was either fixed in $4 \%$ buffered formalin or in $96^{\circ}$ alcohol. All macrofaunal organisms (> $250 \mu \mathrm{m}$ ) were identified to the lowest taxonomic level possible. Although not all organisms could be determined to the species level, all taxonomic levels were incorporated in the diversity calculations - even the undetermined taxa because they were considered different from the other identified species. Those identified at the higher taxonomic levels may harbor more than one species, leading to an underestimation of global diversity. Also, meiofaunal identification was limited to higher taxonomical groups. Technical problems with the suction sampler device, that is more efficient to collect meiofauna, led us to remove RB3 sample from the statistical analyses that include this faunal compartment.

For each sampled assemblage, the surface area was estimated using the ImageJC image analysis software on images taken before and after sampling (according to Sarrazin et al., 1997). To facilitate image calibration, calibrated targets (Casimir) were deployed in the vicinity of each assemblage. These targets had visible $7 \times 7 \mathrm{~mm}$ checkerboards that were used as size references in the image (Figure 2). Each sampled area was analyzed six times by different observers to reduce error resulting from on-screen tracing. Even though it is still the most common way to estimate surface area for hard substratum samples, this method does not account for the relief nor the thickness of the faunal coverage, inducing a bias in density estimates. Because of the difficulty of access and the steepness of the slopes on the 
RB edifices, no calibrated target could be deployed. Surfaces were estimated using the ROV temperature cannula as a calibrating device on the image, and the samples are thus considered as semi-quantitative. In addition, surface from RB1 could not be estimated, and only the raw abundance of the fauna within this sample was used. Mussel sizes were estimated using the same protocol and assemblages were categorized following the mean mussel size described in Cuvelier et al. (2011a): large (> $6 \mathrm{~cm})$, medium $(2-6 \mathrm{~cm})$, and small $(<$ $2 \mathrm{~cm})$.

\section{Data analyses}

All analyses were performed in the $R$ environment ( $R$ core team 2018). Environmental data were used in a principal component analysis (PCA) to compare conditions within the mussel assemblages in all samples. Spearman correlations between variables were confirmed through a correlation test using permutations.

The faunal composition in the mussel assemblages was compared among edifices and fields using a PCA on Hellinger-transformed density data. Similarity tests assessed whether similarities were higher among edifices or among vent fields. The $\mathrm{R}$ statistic can be interpreted like a correlation, with $\mathrm{R}=0$ meaning that similarity is low among samples from the same group, and $\mathrm{R}=1$ indicating a strong correlation.

The taxonomic richness of the faunal assemblages was compared by analyzing the taxa shared among edifices and fields, and through rarefaction curves of meio- and macrofauna. Diversity indices were studied using the Rényi's generalized entropy. It is an information- 
$\alpha:$

$$
\mathrm{H}^{\alpha}=\frac{1}{1-\alpha}\left(\ln \sum_{\mathrm{i}=1}^{S} p_{1}{ }^{\alpha}\right.
$$

where $S$ is the total number of taxa, pi the relative abundance of taxon $i$, and $\alpha \geq 0$ and $\alpha \neq 1$.

The effective number of species for Rényi's entropy, $D^{\alpha}=\exp \left(H^{\alpha}\right)$. Special cases of Rényi's entropy are well-known indices: for $\alpha=0, D^{\alpha}=S$, the species richness; for $\alpha=1, D^{\alpha}$ is the exponential of Shannon's index; for $\alpha=2, H^{\alpha}$ is linked to Simpson's measures: for $\alpha \rightarrow \infty, H^{\alpha}$ is the Barger-Parker dominance index. Results from the profile of each taxon were used in a PCA to better understand differences between samples. As for the species composition analysis, a similarity analysis was conducted to compare samples from the same edifice or the same field.

To understand how environmental conditions are linked to species composition, canonical redundancy analysis was used on Hellinger-transformed meio- and macrofaunal densities. It was supplemented with a variation partitioning analysis to determine the relative effects of endogenous (minimum, maximum, mean and standard deviations of temperature as proxies for hydrothermal influence) and exogenous variables (depth, end-member temperatures, distance to MG). The same analysis was repeated for samples from the LS vent field only to examine intra-field structuring factors. For this analysis, distance to MG was replaced by distance to Montségur edifice (Dist_MO).

\section{Results}


All results are divided into the "local scale" which represent intra-field data from LS ( $n=15$ samples on four edifices) and "regional scale" which represent inter-field data from all collected samples and compares vent fields ( $n=21$ samples from seven edifices).

\section{Environmental conditions}

Local scale: The mean temperature measured on CY (edifice Cypress from Lucky Strike) varied between 5.6 and $6.2^{\circ} \mathrm{C}$ (Table 1). Maximum and minimum temperatures were found on $\mathrm{CY} 3$ with $10.0^{\circ} \mathrm{C}$ and $4.9^{\circ} \mathrm{C}$, respectively. The mean temperature measured on $\mathrm{Y} 3$ (edifice Y3 from Lucky Strike) varied between 6.8 and $7.9^{\circ} \mathrm{C}$ (Table 1). Maximum and minimum temperatures were found on $\mathrm{Y} 32$ and $\mathrm{Y} 33$ with $11.8^{\circ} \mathrm{C}$ and $4.5^{\circ} \mathrm{C}$, respectively. $4.5^{\circ} \mathrm{C}$ corresponds to the bottom seawater temperature at the vent field. The mean temperature measured on TE (edifice Eiffel Tower from Lucky Strike, 2011 \& 2013) varied between 5.3 and $11.2^{\circ} \mathrm{C}$ (Table 1). Maximum temperatures were found on TE1 and TE5 with $29.7^{\circ} \mathrm{C}$ and minimum was observed on TE3 \& TE6 with $4.5^{\circ} \mathrm{C}$. On MO (edifice Montségur from Lucky Strike), the mean temperature measured varied between 5.1 and $7.8^{\circ} \mathrm{C}$ (Table 1). Maximum temperature was found on $\mathrm{MO} 2$ with $14.2^{\circ} \mathrm{C}$ and minimum was $4.5^{\circ} \mathrm{C}$ in all samples. $\mathrm{H}_{2} \mathrm{~S}$ concentrations measured above various mussel assemblages of the vent field varied between 0.99 and $9.96 \mu \mathrm{M} . \mathrm{Y} 3$ exhibited the lowest values while MO had the highest (Table S1).

Regional scale: Menez Gwen. The mean temperature measured on Atos 10 ranged from 9.6 to $10.3{ }^{\circ} \mathrm{C}$ (Table 1). Maximum temperature among the three samples was $25.5^{\circ} \mathrm{C}$ on $\mathrm{MG} 2$, and the minimum temperature was $8.8^{\circ} \mathrm{C}$ in all samples. This minimal value also corresponds 
to the bottom seawater temperature of the vent field. Rainbow. The mean temperature estimated on Rainbow was $7.8^{\circ} \mathrm{C}$, with a maximum of $39.7^{\circ} \mathrm{C}$ and a minimum of $3.9^{\circ} \mathrm{C}$ (Table 1). In general, temperature variations (standard deviations) were higher on RB. Depths of the sampling sites varied from $826 \mathrm{~m}$ on MG to $2274 \mathrm{~m}$ on RB, with those of the LS vent field being located at intermediate depths ranging from 1690 to $1740 \mathrm{~m}$ (Table 1). Temperatures of the end-member fluids varied from $284^{\circ} \mathrm{C}$ on MG, 304 to $325^{\circ} \mathrm{C}$ on $\mathrm{LS}$ and $362^{\circ} \mathrm{C}$ on $\mathrm{RB}$ (Table 1). To complete the dataset, a series of environmental conditions were evaluated in one mussel assemblage per vent field ( $n=3$, Table S1). The mussel assemblage from MG was characterized by the lowest concentrations of $\mathrm{CH}_{4}$ and intermediate Fe concentrations. $\mathrm{H}_{2} \mathrm{~S}$ concentrations varied between 33 and $51 \mu \mathrm{M}$, higher than those observed for LS. The LS assemblage was characterized by the lowest concentrations of dissolved Fe. Overall, the $\mathrm{H}_{2} \mathrm{~S}$ concentrations in various LS mussel assemblages varied between 0.99-9.96 $\mu \mathrm{M}, \mathrm{Y} 3$ exhibiting the lowest values and MO the highest. Finally, the RB assemblage was characterized by the highest concentrations of $\mathrm{CH}_{4}$ and dissolved $\mathrm{Fe}$ and intermediate to elevated range of $\mathrm{H}_{2} \mathrm{~S}$ (based on extrapolation). In $L S$ and $M G$, the mean and maximum extrapolated $\mathrm{H}_{2} \mathrm{~S}$ concentrations are higher than the ones obtained from in situ analyses (Table S1). In addition, patterns of $\mathrm{H}_{2} \mathrm{~S}$ variations in the mussel assemblages from the three fields are not consistent between the different approaches (measured versus extrapolated; Table S1). In the absence of in situ $\mathrm{H}_{2} \mathrm{~S}$ data, only site by site extrapolation of $\mathrm{H}_{2} \mathrm{~S}$ from the specific dilution curve of each edifice could help evaluating major trends in environmental conditions above mussel assemblages. In the meantime, extrapolated values of $\mathrm{H}_{2} \mathrm{~S}$ are not reliable to explain the observed patterns. Finally, bottom water temperatures varied between the three fields, ranging from $3.5^{\circ} \mathrm{C}$ at $\mathrm{RB}$ to $8.8^{\circ} \mathrm{C}$ at MG (Table S1). 
The PCA on available environmental variables explained $93 \%$ of the total variance observed

between the samples on its first two dimensions (Figure 3). As shown in Figure 3, the first axis, which explains $56 \%$ of the variance, was positively correlated with exogenous factors (distance to MG, end-member temperature, field depth) and negatively correlated with minimal temperatures (endogenous factor). Many of these variables were significantly correlated with each other, positively or negatively (Table 2). The second axis was positively correlated with endogenous factors (mean, maximum and standard deviations of temperature). It also showed a good regression of mussel size $\left(R^{2}=0.70, p=6.7910^{-5}\right)$, with large mussel assemblages found in the upper part of the PCA and small ones on the lower part. Sample CY3 is an exception, because the assemblage was composed of large mussels, but its environmental conditions were more similar to those found in small and medium mussel assemblages. The third axis did not significantly explain variance in environmental conditions ( $p>0.05)$. The presence or absence of microbial mats did not show any significant pattern in the PCA, although samples with microbial mats (TE3, TE4, Y31, MO3) were all situated in the lower part of the diagram, in conjunction with lower temperature conditions (Figure 3).

This PCA thus divided samples according to exogenous variables on the first axis and endogenous variables (local temperatures) on the second. Four groups are visually distinct on the PCA: 1) MG samples with higher minimal temperatures, lower end-member temperatures and lower depth; 2) The only RB sample lies opposite to MG on the first axis, with higher maximal temperatures, standard deviation and end-member temperatures, the 
greatest depth and greatest distance to MG, and lower minimal temperatures; 3) TE5 and TE1 (large mussels) were characterized by intermediate exogenous conditions, but higher maximal and standard deviation of temperatures; 4) all other samples from LS were characterized by intermediate exogenous and endogenous conditions.

\section{Surface areas and faunal densities}

Local scale: On LS, the surfaces sampled varied from 169 to $318 \mathrm{~cm}^{2}$ on CY, from 169 to 279 $\mathrm{cm}^{2}$ on $\mathrm{Y} 3$, from 122 to $856 \mathrm{~cm}^{2}$ on TE and from 175 to $373 \mathrm{~cm}^{2}$ on MO (Table 1). The area sampled on TE1 was substantially larger than the others. Mussel densities within the samples ranged from 2209 ind. $/ \mathrm{m}^{2}$ on TE1 to 46092 ind. $/ \mathrm{m}^{2}$ on $\mathrm{CY} 1$. Of all the LS assemblages, one-fifth (i.e. 3 out of 15) were composed of large mussels (CY2, TE1 and TE5), and nearly half (7 out of 15 ) were medium-sized mussels, leaving one-third of small mussels (Table 1). The three largest densities of associated fauna were found in medium-sized assemblages, and two of them were situated on MO edifice: MO2 with $100380 \mathrm{ind} . / \mathrm{m}^{2}$, MO1 with 126437 ind./m² and TE4 with 232414 ind./m². The three lowest densities of associated fauna were found in medium to larger-sized mussel assemblages: one among the medium-sized mussels (Y32) with 23067 ind./ $\mathrm{m}^{2}$ and two among the large-sized assemblages (TE1: 18,678 ind. $\mathrm{m}^{2}$, and CY3: 17581 ind./m², Table 1). Most of the assemblages sampled were devoid of visible microbial mats with the exception of four of them (Y31, TE3, TE4, MO3, Table 1).

Regional scale: The surface areas sampled on MG were similar to those on LS, ranging from $288 \mathrm{~cm}^{2}$ on MG1 to $431 \mathrm{~cm}^{2}$ on MG2. RB showed more variable surface areas due to sampling difficulties on its steep chimney walls. The lowest was RB3 with $375 \pm 30 \mathrm{~cm}^{2}$ and 
351

352

353

354

355

the highest RB2 with $992 \pm 36 \mathrm{~cm}^{2}$. Sampled surface area on RB1 could not be estimated due to issues with the suction sampler. Mussel assemblages taken from MG and RB were all composed of large mussels, in higher densities on MG (from 1555 ind./m² on MG2 to 2427 ind./m² on MG1, Table 1), than on RB (from 756 ind./m² on RB2 to 1574 ind./ $/ \mathrm{m}^{2}$ on RB3). These densities were lower than those found on the LS assemblages. Faunal densities of the associated fauna on MG were in the lower end of those found at LS, with values varying from 11487 ind./m² on MG2 to 50675 ind./m² on MG3. The two RB samples available displayed both the lowest (RB2: 8002 ind./m²) and the second highest densities in this study (187 795 ind./ $\mathrm{m}^{2}$ on RB3, Table 1). Microbial mats were only found at LS. Overall, there was a significant relationship between mussel size and the density of associated fauna (F-value $=$ 13.49, $p$-value $=0.00031)$, the highest densities being found in small-sized mussel assemblages.

\section{Species composition}

A total of 10 meio- and 33 macrofaunal taxa were identified in our samples: 36 taxa were identified from LS vent field, 13 from MG, and 21 from RB (Figure 4a).

Local scale: The composition of species among the assemblages of the four LS edifices varied. On Cypress (CY), the samples were dominated by macrofauna (from $52 \%$ of the relative faunal abundance in $\mathrm{CY} 1$ to $72 \%$ in $\mathrm{CY} 3$ ). Among the three macrofaunal samples, two were dominated by the species trio Branchipolynoe seepensis, Amathys lutzi and Protolira valvatoides, all considered as indicator species of $B$. azoricus mussel assemblages (Table 3). The third sample was also dominated by $A$. lutzi and B. seepensis, but had high abundances 
of Mirocaris fortunata. Copepods (+ nauplii) largely dominated the meiofaunal compartment in the three samples representing $>68 \%$ of the abundance (Table 4 ). Nematodes were the second dominant taxa closely followed by ostracods in CY3 (Table 4). On Y3, two samples were dominated by macrofauna ( $81 \%$ of the relative faunal abundance in $\mathrm{Y} 31,62 \%$ in $\mathrm{Y} 32$ ) and Y33 abundance was largely dominated by meiofauna (>80\%). Among the three macrofaunal samples on $Y 3$, two were largely (>60\%) dominated by amphipods, followed by either B. seepensis or M. fortunata (Table 3). The third sample (Y33) was dominated by $B$. seepensis, followed by $M$. fortunata and $A$. lutzi. Copepods largely dominated the meiofaunal compartment in two $Y 3$ samples (Y31 \& Y33), but nematodes dominated $Y 32$ (Table 4). Ostracods were the second dominant taxa in Y31 \& Y33 and copepods were the second dominant taxa Y32 (Table 4). Four of the six samples (2011 \& 2013) taken on Eiffel Tower (TE) were dominated by meiofauna ( $67 \%$ of the total abundance in TE1, $87 \%$ in TE3, 95\% in TE4, 84\% in TE5). The two other samples (TE2 and TE6) were dominated by macrofauna representing respectively $86 \%$ and $84 \%$ of the total faunal abundance. In 2011 , the three macrofaunal samples from TE were dominated by different taxa (Table 3). TE1 was dominated by Amphipoda, followed by M. fortunata and B. seepensis. TE2 was dominated by two gastropod species: P. valvatoides and Lepetodrilus atlanticus followed by B. seepensis. TE3 was largely dominated by polychaetes, starting with dorvilleids followed by $B$. seepensis and $A$. lutzi. In 2011, the meiofaunal compartment was dominated by three different taxa: copepods in TE1, nematodes and tanaids in TE2 and ostracods and nematodes in TE3 (Table 4). In 2013 , all three macrofaunal samples were dominated by $B$. seepensis followed by either A. lutzi (TE4 and TE5) or P. valvatoides (TE6, Table 3). The third dominant taxa in TE5 were $M$. fortunata and nemerteans. Overall, B. seepensis was among the three dominant species in all six TE samples. The meiofaunal compartment was dominated by nematodes 
followed by copepods in TE4 and a trio of copepods, nematodes, ostracods in TE5 and TE6 (Table 4). All three Montségur samples were dominated by meiofauna representing respectively $74 \%, 86 \%$, and $78 \%$ of the total faunal abundance. The MO1 macrofaunal sample was dominated by $P$. valvatoides, A. lutzi and B. seepensis and $\mathrm{MO} 2$ was dominated by two of the same species ( $A$. lutzi and B. seepensis) as well as by Pseudorimula midatlantica. $\mathrm{MO} 3$ was dominated by $B$. seepensis followed by dorvilleid polychaetes and aplacophorans (Table 3). Nematodes were the most dominant taxa of $\mathrm{MO} 1$ and $\mathrm{MO3}$, and MO2 was dominated by a trio of copepods, nematodes and ostracods (Table 4).

Regional scale: MG samples were dominated by meiofauna (from $57 \%$ of the relative faunal abundance in MG2 to 76\% in MG3), mostly by copepods (MG1 \& MG2) and foraminifers (MG3; Table 4). Accordingly, nauplii had their highest abundances in MG1 and MG2. The three macrofaunal samples were largely dominated by $P$. valvatoides (Table 3 ). The second most dominant species was the gastropod L. atlanticus. The two RB samples for which we identified macro- and meiofauna had different abundance patterns: RB1 was dominated by macrofauna and RB2 was dominated by meiofauna representing respectively $78 \%$ and $97 \%$ of the total faunal abundance. The three macrofaunal samples were dominated by $A$. lutzi (Table 3). The second most dominant macrofaunal species was L. atlanticus on RB1, $M$. fortunata on RB2 and spionid polychaetes on RB3. M. fortunata was the third dominant species on RB1, spionids and nemerteans on RB2 while on RB3, $P$. valvatoides and $P$. midatlantica shared the dominance at the third position (Table 3). Nematodes largely dominated the RB meiofaunal sample for which we have data (RB1 \& RB2) followed by copepods on RB1 (Table 4). 
422 The PCA explained $32.5 \%$ of species composition on two dimensions, but the second, third

423 and fourth dimensions explained approximately the same amount of variance with $13.6 \%$,

424

425

426

427

428

429

430

431

432

433

434

435

$11.4 \%$ and $11.2 \%$, respectively (Figure 5). Only the three first dimensions were significant.

The first axis constituted a suitable regression for assemblage mussel size $\left(R^{2}=0.38, p=0.022\right)$. Assemblages of small mussels were significantly associated with the right $(p=0.015)$ and large ones with the left $(p=0.031)$ side of the PCA. The first axis was positively correlated with Halacaridae, Tanaidacea, Sericosura heteroscela, A. lutzi, B. seepensis, Cirratulidae, Lirapex costellata, Glyceridae, Spionidae, Sipuncula, Laeviphitus desbruyeresi, L. atlanticus, Pseudorimula midatlantica, P. valvatoides, Xylodiscula analoga and negatively correlated with Copepoda.

The second axis made for a good regression for the different edifices $\left(R^{2}=0.73, p=0.006\right), M G$ being the only site visually separated from the others. MG was significantly associated with the lower part of the figure (Figure $5, p=0.0003)$, and Cypress the upper part $(p=0.02)$. The second axis divided the PCA into two parts: i) in the top part: samples with higher densities of A. lutzi, Ostracoda, P. smaragdina, M. fortunata, L. jouinae, Branchinotogluma sp., B. seepensis and S. mesatlantica; ii) in the lower part: samples with higher densities of $P$. valvatoides, L. atlanticus, Foraminifera and nauplii.

The third axis was positively correlated with Aplacophora, Glyceridae, Xylodiscula analoga, Lurifax vitreus, Hesionidae, nauplii, and Turbellaria. Cypress was significantly associated with high densities of these species. However, the third axis was also negatively correlated with Sipuncula and Cirratulidae. 
On LS, 16 taxa were shared between all studied edifices and 17 were shared between two or more edifices. Five taxa were observed on only one edifice (Figure 4B): Hesionidae was found only on $\mathrm{CY}$, Thalycrocuma sarradini was found only on $\mathrm{MO}$ and Sipuncula, Demospongiae and Levensteiniella iris were found only on TE. At the regional scale, only six taxa were shared between all fields: P. valvatoides, L. atlanticus, M. fortunata, Amphipoda, Copepoda and Foraminifera (Figure 4A), but only three of them (L. atlanticus, M. fortunata, Copepoda) were present in all samples. LS shared 16 taxa with RB and 11 with MG. 15 taxa out of the 36 observed at LS were absent from the two other vent fields. MG had two unique taxa: Isopoda and Euphausiidae and RB had 5: Rimicaris exoculata, Alvinocaris markensis, Rimicaris chacei, Archinome and Ophiura (Figure 4A).

\section{Faunal diversity}

Taxon accumulation curves- Macrofaunal rarefaction curves (supplementary material, Figure S.2a.) display a gradient of diversity between all samples, with samples from the same edifice showing similar curves. None of the curves reached an asymptote, suggesting that not all macrofaunal taxa have been sampled yet. However, the final slopes tend to decrease, suggesting that the most common taxa have been collected. Meiofaunal rarefaction curves (supplementary material, Figure S.2b.) are more difficult to interpret due to the low identification level and low abundances in some samples. The poor diversity of meiofauna is due to the low taxonomic resolution attained for copepods and nematodes in particular.

Local scale: On LS, Montségur showed the highest macrofaunal diversity values, followed by TE 2013 and CY (supplementary material, Figure S.2a.). Y3 and TE 2011 samples displayed 
intermediate diversities, with Y31, Y32, TE1 and TE3 having lower expected richness than the

468

469

470

471

472

473

474

475

476

477

478

479

480

481

482

483

484

485

486

487

others. For the meiofauna, MO1 and MO3 samples and most TE samples (TE2, TE3, TE4, TE5, TE6) showed high expected richness (supplementary material, Figure S.2b.)

Regional scale: MG showed the lowest expected macrofaunal richness and evenness and RB displayed varying levels of diversity, with high values in RB1, similar to TE and MO expected richness, and low values on RB3 in the range of those found in Y3 samples (supplementary material, Figure S.2a.). RB2 showed low expected meiofaunal richness, and reached an asymptote, indicating that all meiofaunal taxa (at this level of identification) have probably been sampled (supplementary material, Figure S.2b.).

\section{Renyi's entropy index}

Local scale: Renyi's profile based on meio- and macrofaunal densities (supplementary material, Figure S.3.) exhibited higher taxonomic richness for $\mathrm{MO}$ and TE samples and Y31 show the lowest number of taxa. TE samples had the highest evenness for both years.

Regional scale: MG samples had low taxonomic richness and diversity equivalent to Y31 (supplementary material, Figure S.3.). RB diversity indices (Alpha= 1 and 2) were much lower than the other samples although its taxonomic richness was higher than that on MG and two samples from Y3 (Y31 and Y32). 
A PCA should not include correlated variables. Therefore, as values of entropy for $\alpha=2$ and $\alpha$

489

490

491

492

493

494

495

496

497

498

499

500

501

502

503

504

505

506

507

508

509

$=\rightarrow \infty$ were strongly correlated (corr $=0.9, p<1.10^{-5}$ ), only $\alpha=0,1$ and $\rightarrow \infty$ were used in the

PCA (Figure 6). The total explained variation of the PCA with Renyi's entropy index was 93\%,

with $73 \%$ from the first axis, which was correlated with all three variables (1: corr $=0.92$, $p=1.9 .10-8 ; \rightarrow \infty:$ corr $=0.88, p=6.5 .10-7 ; 0:$ corr $=0.75, p=2.2 .10-4$, Figure 6$)$. The first axis was

a good regression to explain variability between the edifices $\left(R^{2}=0.62, p=0.04\right)$, with Thermitière (RB2 and RB3) being significantly associated with the left side of the PCA $(p=0.036)$ representing low diversity. Large mussel assemblages were also associated significantly ( $p=0.033$ ) with this part of the PCA (Figure 6). The second axis was only significantly correlated with Renyi's entropy for alpha $=0$ (corr $=0.66, p=0.002$ ), and differentiated samples linked to high specific richness in the upper part of the PCA, from those with low specific richness in the lower part. MO is significantly correlated with high richness levels (corr=0.88, $\mathrm{p}=0.017$ ). All samples from MG show low diversity and richness. RB showed even lower diversity, but higher species richness. LS edifices displayed a large variety of diversity indexes, with MO and four out of six samples from TE having the highest values. Y3 had the lowest diversity and richness of all four LS edifices (Figure 6).

\section{Similarity in composition and diversity}

Composition: The analysis of similarity was not significant for intra-edifice comparisons $(R=0.05, p=0.35)$, indicating that the faunal composition of a sample from a single edifice is not more similar with another sample of that edifice than a sample from another edifice in the vent field. However, the similarity analysis was significant for intra-field comparisons 
$(R=0.82, p=0.001)$ indicating that the faunal composition of a sample from the same vent

field share more similarities than with a sample from the two other vent fields.

Diversity: The similarity of the diversity indices from the same edifice was not significant $(R=0.09, p=0.22)$ indicating that there is no more similarity within a single edifice than between different edifices of the same vent field. The similarity of the diversity indices of samples from a single vent field was significantly higher than the similarity of samples from different vent fields $(R=0.68, p=0.001)$.

Linking faunal assemblage structure with the environment

The two first dimensions of the environmental PCA (Figure 3) were used to constrain the

RDA. The realized RDA accounted for $52 \%$ (adjusted $R^{2}: 33 \%$ ) of the variability in species

Dim.1. Dim. 2 is linked to endogenous environmental factors such as minimal, mean, maximal and standard deviation values of temperatures in the assemblages, but Dim. 1 is linked to exogenous factors (depth, end member temperature and distance to MG vent field). Samples were distributed according to their vent fields along Dim.1 with RB and MG opposing each other: MG as the shallow vent field with low end-member temperatures and high densities of Foraminifera, Copepoda and nauplii versus the deeper RB vent field, further away from MG and characterized by higher end-member temperatures and higher densities of nematodes. All LS samples had intermediate coordinates on this dimension and varied

530 with respect to the differing densities of $A$. lutzi and $B$. seepensis, which appear to be more abundant in small-sized mussel assemblages. 
533

534

535

536

537

538

539

540

541

542

543

544

545

546

547

548

549

550

551

552

553

554

Variation partitioning

Local scale: Variation partitioning with all variable outputs was not significant. However, when performing forward selection on each subset, no endogenous variables yielded a $p$ value below 0.05 (lowest $p=0.21$ ) and the only exogenous variable to have a significant effect on species density was the distance to Montségur $(p=0.03)$.

Regional scale: Given that endogenous and exogenous variables were highly correlated (Table 3), variation partitioning analyses were performed on subset of the best explanatory environmental variables according to a partial forward selection. Forward selection of endogenous variables to explain species Hellinger-transformed densities led to the selection of only one variable, Min_T (adjusted $\mathrm{R}^{2}: 0.19, \mathrm{p}=1.0 .10^{-4}$ ), and the same process on exogenous variables revealed two explanatory factors: depth (adjusted $R^{2}: 0.18, p=0.0002$ ) and distance to $M G$ (cumulative adjusted $R^{2}=0.25, p=0.0099$ ). Variation partitioning with these three parameters results in an adjusted $\mathrm{R}^{2}$ of 0.065 for the endogenous variable alone, 0.13 for the exogenous ones, and 0.12 for their joint effect, for a total explained variance of $\mathrm{R}^{2}$ adjusted $=0.32$

\section{Discussion}

This study aimed at assessing inter- and intra-field variations in community composition within the dominant $B$. azoricus assemblages of three northern MAR vent fields, thus expanding on the few similar studies performed on this ridge section (Van Dover et al., 1995; Desbruyères et al., 2000; 2001; Goroslavskaya and Galkin, 2011; Fabri et al., 2011; Rybakova 
555

556

557

558

559

560

561

562

563

564

565

566

567

568

569

570

571

572

573

574

575

576

577

and Galkin, 2015). Although our data set is strongly asymmetrical and includes uneven sampling effort between vent fields (low number of edifices sampled for MG and RB), it represents one of the most comprehensive integrated faunal and environmental datasets obtained across hydrothermal vent fields. Its limitations are mainly due to the technical difficulties of gathering a comprehensive set of environmental data, without gaps. This was overcome by using temperature as a proxy of hydrothermal fluid inputs. Moreover, undertaking quantitative sampling on hard substrata in the deep sea using a submersible, especially on the very steep vertical surfaces of hydrothermal edifices remains a challenge. Another limitation is due to the fact that some faunal samples were taken in different years. This does not affect our field comparison because the three fields were explored during the same year, but may affect comparison at the field scale.

Taxa associated with B. azoricus were compared across four edifices (Cypress, Y3, Eiffel Tower, Montségur) from the Lucky Strike, one active site from the Menez Gwen (Atos 10) and two edifices from the Rainbow (France 5, Thermitière) vent fields. These comparisons of associated species hosted by the same engineer species in different vent fields were used to assess diversity patterns that may (or may not) be explained by the intrinsic characteristics of the fields. Overall, a total of 43 meio- and macrofaunal taxa were identified in our 21 samples. Differences in community structure and diversity between different edifices of the same field (LS) and between vent fields (MG, LS, RB) were evaluated. The influence of environmental variables on the observed faunal patterns was tested and the relative contribution of endogenous versus exogenous factors evaluated. 


\section{Variability in mussel assemblages from the same vent field: the case of Lucky Strike}

The LS vent field has been extensively studied over the past 25 years. It harbors more than 20 active hydrothermal edifices that are colonized by dense mussel assemblages (Desbruyères et al., 2001; Ondreas et al., 2009). This vent field is part of the EMSO-Azores observatory (Cannat et al., 2011), where recurrent cruises in the area occur, providing several opportunities for sample acquisition and chemical characterization. Thus, LS represented an ideal setting to study intra-field variations in community composition. A total of 36 meio- and macrofaunal taxa were found at LS in the present study. Most samples were composed of medium-sized mussels, which harbored the largest densities of associated fauna. Mussel assemblages occur at mean temperatures varying from 5.1 to $11.2^{\circ} \mathrm{C}$, with minimum values of $4.5^{\circ} \mathrm{C}$ and maximum values of $29.7^{\circ} \mathrm{C}$. These mean temperatures are slightly warmer than the range $\left(4.8\right.$ to $\left.8.8^{\circ} \mathrm{C}\right)$ found by Husson et al. (2017) for TE assemblages, highlighting the high variability of environmental conditions in both space and time.

Among the 36 identified taxa, $42 \%$ were shared between all studied edifices and $47 \%$ were shared between two or more edifices. Five taxa (14\%) were specific to a single edifice and the same amount of taxa were recurrent across all sites and samples. In fact, Branchipolynoe seepensis, Amathys lutzi, Mirocaris fortunata and Lepetodrilus atlanticus were present in all LS samples and Protolira valvatoides was present in all samples but one. The latter dominated two samples and was the second dominant species in two others. B. seepensis dominated more than half of the macrofaunal samples and was the second dominant species in most of the others. A. lutzi was the second dominant species in $40 \%$ of the samples. The three most dominant species (B. seepensis, A. lutzi, P. valvatoides) were 
602

603

604

605

606

607

608

609

610

611

612

613

614

615

616

617

618

619

620

621

622

623

624

previously identified as indicator species of cold microhabitats on the Eiffel Tower edifice (Sarrazin et al., 2015) along with L. atlanticus. In the present study, the latter was present in all samples, but generally in low abundance. These results suggest high connectivity at the scale of LS. Similarity indices of taxonomic composition support this assumption because they indicate a higher resemblance at the vent-field scale than at the edifice scale. Further observations suggest high connectivity at the vent-field scale. The presence of polychaete larvae and adults as well as juveniles of gastropods, amphipods and copepods in traps deployed at large distances $(1000 \mathrm{~m})$ from TE supports the dispersal capabilities of some species at the field scale (Lesongeur et al., 2014). In addition, the settlement of vent meioand macrofauna on substrata deployed away from any hydrothermal activity also suggests good dispersal capabilities and potentially high connectivity between LS edifices (Gaudron et al., 2010; Zeppilli et al., 2015; Plum et al., 2016, Baldrighi et al., 2018; Alfaro-Lucas et al. submitted).

Despite the observed compositional similarity, faunal densities and thus evenness and diversity appear to vary strongly from one edifice to the other at LS. Densities varied by four orders of magnitude within the same vent field. On some edifices, densities were mainly dominated by a few species. In fact, over $60 \%$ of the LS samples were dominated (representing at least $45 \%$ of the relative densities) by a single macrofaunal taxa, either $B$. seepensis, A. lutzi, amphipods or dorvilleids. Dominance by a small number of extremely abundant species is characteristic of vent distributional patterns (Sarrazin and Juniper, 1999; Van Dover and Trask, 2000; Van Dover, 2002; Matabos et al., 2011; Sarrazin et al., 2015). These differences in relative abundances and densities result in low similarities in diversity 
625 profiles among the LS edifices. A gradient of diversity between all samples was observed,

626 with samples from the same edifice showing similar curves. Rarefaction curves suggest that

627 the most common macrofaunal taxa have been collected, which is supported by the fact that

628 a large fraction ( 63\%) of the macrofauna previously collected at TE (Sarrazin et al., 2015)

629 was identified. Our meiofaunal sampling is more difficult to interpret. Indeed, a much lower

630 proportion ( $30 \%$ ) of the meiofauna previously identified (Sarrazin et al. 2015 ) was sampled

631 in the present study, in part due to the low taxonomic resolution attained for the meiofauna.

632 While 13 nematode and 8 copepod taxa had been identified in previous TE samples (Sarrazin

633 et al., 2015), they were only identified at the subclass or phylum level in this study. 634 Therefore, the richness of meiofauna is expected to be much higher. Accordingly, 635 preliminary results on substrata deployed at the scale of the whole vent field report over 38 636 meiofaunal species (Alfaro-Lucas et al. submitted). The distribution pattern observed for the 637 meiofaunal groups in the mussel assemblages show an alternating dominance between 638 copepods and nematodes on all edifices, with the exception of one sample that was 639 dominated by ostracods. In the Sarrazin et al. (2015) study, most samples were dominated 640 by nematodes and the only sample dominated by copepods was considered as an 641 intermediate habitat in terms of abiotic conditions. To conclude, despite the apparent 642 differences in diversity between edifices, our analyses show that samples from a single 643 edifice are not more similar than samples from other edifices in the vent field, suggesting 644 that Lucky Strike enjoys high connectivity.

646 Among the four LS edifices, MO showed a higher diversity profile, followed by TE. This strong 647 similarity between the two can be explained by their very short geographical distance ( $\sim 0$ 
$\mathrm{m})$ as well as their belonging to similar chemistry domains (see below). These two edifices were the only ones to harbor dorvilleids and tanaids. $\mathrm{Y} 3$ and $\mathrm{CY}$, on the contrary, had much lower diversity profiles. Amphipods and copepods dominated the former but polychaetes and copepods were the most abundant taxa in the latter. Desbruyères et al. (2001) also reported the absence of noticeable differences in species composition at the scale of LS, with the exception of Y3. Causes for these disparities can be attributed to the vent geochemical context. According to the sizes of visible hydrothermal deposits, our four active sites appear to be young (Ondreas et al., 2009). They may have formed after faulting, creating new pathways for fluids in the volcaniclastic material deposited over the area (Fouquet et al., 1998). They were then cemented by silica, which precipitated from cooler and diffuse fluid circulation. Despite the observed geological similarity, a chemical gradient is observed in end-member fluids with a decrease in chlorinity and an increase in metal concentrations from CY,Y3, TE/MO (Chavagnac et al., 2018). These differences may locally affect the colonization of vent species according to their levels of tolerance. Essential metals ( $\mathrm{Co}, \mathrm{Cu}, \mathrm{Fe}, \mathrm{Mn}, \mathrm{Ni}, \mathrm{Zn}$ ) can be toxic if present in excess, and non-essential metals such as $\mathrm{Ag}$ and $\mathrm{Cd}$ are considered toxic (Langston et al., 1998). However, their role in shaping vent species distribution is unknown because these habitats are naturally enriched in metals (Sarradin et al. 2009, Cotte et al. in press). In addition to metal concentrations, the role of the reduced chemicals used for chemosynthesis (such as $\mathrm{H}_{2} \mathrm{~S}, \mathrm{CH}_{4}$, iron and hydrogen) is also important to take into account. These reduced chemicals are potential energy sources for vent microorganisms and could be important drivers of species distribution in hydrothermal ecosystems (Sarrazin et al. 1997, 1999, 2015, Gebruk et al., 1997, Sarradin et al., 1998, Luther et al., 2001, Marsh et al., 2012; Sen et al., 2013, Cuvelier et al., 2011a, 2014b; Husson et al. 2017). Higher concentrations of hydrogen sulfide (or other reduced compounds) may 
672

673

674

play a role in sustaining a greater faunal diversity by offering a wider breadth of trophic resources. On the contrary, due to its toxicity, hydrogen sulfide may also negatively influence faunal diversity, resulting in less diverse communities in harsher habitats as it was observed previously (Sarrazin et al. 1999, Gollner et al. 2010).

Studying samples from the same vent field limits the number of environmental explanatory variables that can be used, because depth and end-member fluid temperature are approximately the same for all edifices. Potential explanatory environmental variables included exogenous (distance to Montségur) and endogenous (temperature above the mussel assemblages) factors. At LS, although new evidence argues for the presence of a unique deep fluid source (Pester et al., 2012; Leleu et al., 2015; Chavagnac et al., 2018), four chemistry domains can be distinguished, linked to different upflow patterns through the crust (Barreyre et al., 2012, Leleu et al., 2015, Chavagnac et al., 2018). The first domain includes Crystal, South Crystal, Sintra and Y3; the second Cypress, White Castle and Isabel; and the third Eiffel Tower, Aisics, Cimendeff and Montségur. The fourth domain is represented by the newly discovered edifice Capelinhos, located further away. This site appears to be the most pristine and its fluids are the most representative of the conditions of the reaction zone. These differences may be related to crustal residence time, which is likely related to permeability variations across the hydrothermal upflow zone (Chavagnac et al., 2018). This spatial variability can also be observed in the metal partitioning observed in the early buoyant plume of LS active edifices (Cotte et al in press). These authors found that the chemical signature at each site was similar in the warmest part of the mixing zone (50$150^{\circ} \mathrm{C}$ ) but that metal partitioning was affected by different precipitation and oxidation 
695

696

697

698

699

700

701

702

703

704

705

706

707

708

709

710

711

712

713

714

715

716

processes occurring between 50 and $5^{\circ} \mathrm{C}$. This emphasizes the need to characterize the environmental conditions at the fine scale of the faunal habitats. According to the variance partitioning analysis, the distance to MO (exogenous variable) was the main explanatory variable to explain differences in species densities among the edifices of LS, which could reflect this variability of chemical composition of the fluids across the vent field.

The same variation partitioning analysis revealed the very low explanatory power of endogenous variables chosen by the forward selection process. This is not in agreement with recent studies that have shown a strong structuring effect of temperature on faunal distribution and diversity on TE (e.g. Cuvelier et al., 2011a; Sarrazin et al., 2015; Husson et al. 2017) and elsewhere (Gebruk et al., 1997; Sarrazin et al. 1999; Sarradin et al., 1998; Luther et al., 2001; Marsh et al., 2012; Sen et al., 2013). However, as shown on the PCA on environmental variables, most LS samples showed similar thermal conditions. This similarity may be due to the fact that most samples (80\%) were taken in small- to medium-sized mussel assemblages, primarily associated with cold, less variable habitats (Sarrazin et al., 2015). The weak links between faunal densities and temperature at LS is therefore not surprising, as the range of environmental conditions observed in our faunal samples is narrow. Additional sampling in large-sized mussel assemblages may help to assess whether the thermal gradient has a significant influence on species distribution only at the edifice scale or if it has a similar effect across the vent field. The results of the intra-field similarity analysis showed that there are more similarities between samples from the vent field than between samples from the same edifice, which tends to support the second hypothesis. 
Additional sampling of larger-sized mussels on several LS edifices could help further characterizing the effect of temperature on both mussel size and the composition of associated communities at the vent field scale. Indeed, in the PCA on environmental variables, most large-sized mussel assemblages were associated with warmer habitats. Because of this correlation, size was not explicitly included as an explanatory variable. Previous studies have already established that the thermal range of Bathymodiolus azoricus mussels change with their sizes, each size category occupying a different thermal niche (Cuvelier et al., 2011a; Husson et al., 2017). The link between mussel size and temperature may be explained by the fact that larger individuals, living closer to hydrothermal fluid emissions, may have access to higher concentrations of reduced chemicals and thus benefit from potentially higher energy sources than smaller mussels, leading to higher growth rates. The comparison between the estimated concentrations in $\mathrm{H}_{2} \mathrm{~S}$ from a simple mixing model and those measured in situ on the Menez Gwen and Lucky Strike vent fields (Table S1) shows that the final concentrations above the mussels are overestimated. Such discrepancy can be explained by the consumption of the available reduced chemicals by the local primary production (Cuvelier et al. 2011a). Supplementary sampling of larger size mussels with associated abiotic conditions could help understand whether their proximity to fluid exits favors them with higher potential primary production.

As already observed on $\mathrm{TE}$, the mussel size also influences the composition of associated communities (Sarrazin et al., 2015, Husson et al., 2017). This is linked to the role of Bathymodiolus azoricus as a foundation species (Rybakova and Galkin, 2015), creating threedimensional structures that favor settlement, provide shelter and modulate the 
environment (Van Dover and Trask 2000). However, our results rather show a negative correlation between mussel size and the densities of mussels and that of their associated fauna. Indeed, most of the large-sized mussel samples were associated with a low diversity index, in accordance with previous results from TE (De Busserolles et al., 2009; Cuvelier et al., 2011a, 2014a; Sarrazin et al., 2015). Whether this is linked to the presence of competition between large $B$. azoricus and their associated fauna is unknown. Large-sized mussels may have high metabolic needs that are detrimental to the settlement and survival of other taxa (Dreyer et al., 2005). In addition, the extreme environmental conditions of warmer microhabitats may limit larval recruitment and settlement because fewer species have the physiological tolerance to thrive in such habitats (Husson et al. 2017). Lower diversity in more stressful environmental conditions is a recurrent pattern at vents (Sarrazin and Juniper, 1999; Tsurumi, 2003; Cuvelier et al. 2009, 2011a).

Finally, visible microbial mats were only observed in the LS samples, present in $\sim 25 \%$. These white filamentous mats are dominated by sulfur-oxidizing Beggiatoa species but also harbor diversified microbial communities (Crépeau et al., 2011). These mats develop in areas where hydrothermal fluids mix with seawater, respectively extracting reduced chemicals and oxygen (Nelson et al., 1989; Teske and Nelson, 2006). Results of a recent study have shown that they preferentially colonize areas that are not directly exposed to fluids, but to currents carrying plume material from main smokers (Girard et al., submitted). This hypothesis is supported by our measurements of temperature that were low in the four targeted assemblages. Microbial filaments can serve as food source to many vent organisms and recent trophic studies have revealed that the majority of vent-endemic fauna feed on free- 
764

765

766

767

768

769

770

771

772

773

774

775

776

777

778

779

780

781

782

783

784

785

living microbes (De Busserolles et al. 2009; Govenar 2012; Portail et al., 2018). Interestingly, gastropods were abundant in most mussel assemblages covered with mats, with the exception of $\mathrm{MO2}$, suggesting that the presence of microbial mats may be attractive to grazers. On the contrary, previous observations by Cuvelier et al. (2011a, 2014a) suggested that the presence of an established filamentous microbial cover may inhibit the settlement of gastropods. These observational discrepancies point out the importance of better defining the role of microbial mats on the functioning of vent communities.

This paper showed that Lucky Strike is a good setting to assess intra-field variations of $B$. azoricus assemblages and their associated species. The pool of species in question is largely shared between the edifices, which suggest no obstacles to species dispersion across the vent field. However, differences in relative abundances foster assemblages with varying diversity. These differences may be linked to different physico-chemical conditions in the mussel habitats between the different chemistry domains identified on LS. However, measured endogenous variables (all linked to temperature) do not suffice to explain the beta diversity of mussel assemblages at the field scale. Characterization of other environmental conditions as well as biotic interactions may be the key to assess the observed differences in diversity.

\section{Variability of mussel assemblages from the three studied vent fields}

The present study shows the presence of a "vent field signature" in the communities associated with B. azoricus. Overall, only six taxa were shared between the three fields among which L. atlanticus, M. fortunata and copepods were present in all samples. Faunal 
samples from MG were quite homogeneous and exhibited a distinct profile from the two other vent fields in each analysis, a pattern that has also been observed by other authors (Desbruyères et al. 2001; Rybakova and Galkin, 2015). This shallowest vent field differed from the two others displaying a different taxonomic composition and a dominance of the skeneid gastropod Protolira valvatoides. Environmental conditions at MG include colder endmember temperatures and the highest minimal temperatures over the mussels, probably related to high bottom temperatures.

A parabolic pattern in species diversity with respect to water depth has been observed for many deep-sea taxa (Rex, 1981; Pineda and Caswell, 1998; Chase et al., 1998; Gray, 2001; Gooday et al., 2004; McClain and Etter, 2005) with a peak at intermediate depth (2000 m). This pattern was highlighted in the present study in which LS $(1700 \mathrm{~m})$ shows higher diversity than MG $(850 \mathrm{~m})$ but lower than RB $(2300 \mathrm{~m})$. The same pattern was observed by Desbruyères et al. (2000) between the three vent fields but with a lower taxonomic resolution. These authors suggest that shallower vents harbor less endemic species but a higher number of background taxa. However, this was not observed in our results. Shallower vent fields may also benefit from additional inputs from photosynthetically derived organic carbon as suggested by Bennett et al. (2015). Patterns between diversity and depth among vent ecosystems would be an interesting question to tackle at a global scale. These patterns may be explained by various processes regulating species diversity such as speciation rates, environmental variability, productivity, physiological adaptation, biotic interactions, habitat heterogeneity, and historical evolutionary factors (Ricklefs and Schluter 1993; Gray 2001). The low species diversity at MG may be related to a sampling bias because it has far fewer 
samples than LS. However, the higher expected richness values of RB, which share the same number of samples as MG, do not support this hypothesis. LS, although geographically closer to MG, shares more of its taxa with RB (37\%) than with the former (26\%), a pattern that has also been observed by other authors (Desbruyères et al. 2000, Rybakova and Galkin 2015). One of the observed differences in community structure is linked to the total absence of polychaetes at MG. Polychaetes are among the key taxa contributing to diversity at vents and they are known to colonize a wide variety of niches. Because polychaetes have previously been sampled at this site (Desbruyères et al., 2001, Rybakova and Galkin 2015), we considered a sampling/preservation bias. However, their presence in samples from the two other vent fields, taken during the same cruise using the same protocol, does not support this hypothesis. Alternatively, ecological succession may be operating within the community. For instance, a major change in community composition in MG mussel assemblages may have occurred between 2002-2005 (Rybakova and Galkin, 2015) and 2013 (our study), as highlighted by the switch in dominance from L. atlanticus (59\% of the abundance) to that of $P$. valvatoides ( $>57 \%$ of the abundance) in the latter, changes that may have affected polychaetes. Whether these changes are linked to temporal variations in environmental conditions, to the presence of biological interactions or simply related to spatial variability is unknown. The absence at MG of $B$. seepensis, which is the most abundant polychaete species at LS, has also been reported by other authors (Galkin and Goroslavskaya, 2010).

Overall, the faunal similarity within a single field appears to be more important $(42 \%$ of the species were shared between all edifices at LS) than that between vent fields (only $14 \%$ of 
the species were shared between MG, LS and RB). It is however difficult to determine which are the factors setting MG mussel assemblages apart from those of the other vent fields due to the high correlations between the factors considered here. The fact that MG is located in the upper bathyal $(<1500 \mathrm{~m})$ zone, compared with the lower bathyal $(1500-3000 \mathrm{~m})$ for LS and RB, may be key in explaining the faunal differences observed. The upper bathyal zone is often characterized by distinct physical, geological and biological features and the presence of a different water mass may influence local hydrodynamic processes and also ambient bottom temperatures (Schlitzer, 2000). The role of currents in connectivity between widely spaced hydrothermal fields should also be assessed. Submesoscale $(0.1-10 \mathrm{~km})$ currents are known to play an important role for the dispersal of biogeochemical materials at the ocean surface, but their impact on the dispersion of hydrothermal vent effluents and larvae in the deep ocean are not well understood (Vic et al. 2018). Recent simulations suggest that the connectivity between hydrothermal sites can be increased by submesoscale and tidal currents, which act to spread particles and help them cross topographic barriers (Vic et al. 2018). A persistent current along the flank of the rift valley around $2000 \mathrm{~m}$ was repeatedly observed and confirmed by numerical simulations (Lahaye et al. 2019, see Figure 8), this current may promote larvae transport between the different sites. On the contrary, a relatively strong current at shallower depth trending southeast to northwest near MG probably acts as a strong barrier to larval dispersal with the two other vent fields. In the other direction, larvae would have to be motile enough to ascend to MG or be carried by an upwelling in near bottom layers of the water column (Figure 8). More data on currents and species life-history traits at different spatial scales are still needed to evaluate the fate of particles, including larvae, between the three studied vent fields. 

2001; Mironov et al. 2002; Gebruk \& Mironov 2006; Fabri et al., 2011, Wheeler et al., 2013;

communities of the Atlantic equatorial belt (Cordes et al., 2007; Olu et al., 2010). At vents, depth is known to influence phase separation processes (Charlou et al., 2000; 2002), contributing to modifications in fluid composition (Wetzel and Shock, 2000). Therefore, endmember fluids formed in ultramafic rocks (RB) are characterized by metal-enriched brines, but those from MG and LS are gas-enriched and metal-depleted (Charlou et al., 2000; 2002). Although the physico-chemical compositions of end-member fluids are relatively well known, they are not sufficient to understand the conditions experienced in the diffuse areas colonized by the fauna. For instance, environmental conditions (e.g. reduced compounds and oxygen) in the mixing gradient between cold seawater and hot hydrothermal fluids play

870 a major role in the composition and metabolism of microbial communities, leading to the 871 presence of distinct dominant basal sources and influencing the composition and structure 872 of vent communities (Cerqueira et al., 2017; Portail et al., 2018). In this study, diffuse fluids 873 measured in MG and LS mussel assemblages showed much lower concentrations in methane and iron than those of RB. Metal concentrations in vent fluids may influence the distribution 875 of filter-feeding organisms by forming iron sulfide complexes that limit the availability of hydrogen sulfide (Luther et al., 2001; Charlou et al., 2002) and increase the load in toxic 877 compounds. These potential metal concentrations may explain the restricted spatial 878 distribution of mussel assemblages in the RB high-metal environment (Desbruyères et al. 879 2000). However, despite higher chemical similarities between MG and LS fluid signatures, our study shows more similarity in faunal composition between LS and RB mussel 
881

882

883

884

885

886

887

888

889

890

891

892

893

894

895

896

897

898

899

900

901

902

903

assemblages. Other chemical compounds from the diffuse fluids (not analyzed here, such as ligands as well as organic and inorganic compounds) may influence the availability of sulfur by iron complexation as proposed in Laes et al. (2016) for the TE area over a period of six months (2013-2014). Complementary chemical analysis may help in the understanding of the variation of mussel assemblage over the three sites. In addition, trophic inputs from photosynthetic primary production also vary with respect to depth and may explain differences in community structure between the three fields. However, photosynthesisderived organic matter proved to be a minor energy source in the food webs of our three vent fields (Colaço et al., 2002; 2007; De Busserolles et al., 2009, Portail et al., 2018). Notwithstanding, photosynthetic-OM inputs may indirectly affect vent communities through top-down processes by influencing the distribution of migrant species including predators (Carney, 1994; Carney, 2005; Cordes et al. 2010; Olu et al., 2010; Sahling et al., 2003). It has been hypothesized that the larger number of predators at shallower depths may exert a non-negligible impact on MG assemblages (Rybakova and Galkin, 2015). In addition to depth, field-specific environmental factors such as geological setting and the nature of underlying rocks may also explain the substantial differences observed between fields on the MAR (Desbruyères et al., 2000; 2001; Copley et al., 2007; Fabri et al., 2011). Furthermore, the contribution of basal sources to food webs are influenced by environmental conditions. However, species that were shared between assemblages showed high trophic flexibility, suggesting that the metabolic diversity of basal sources may not be a structuring factor (Portail et al., 2018). These results provide further support to the hypothesis that exogenous rather than endogenous factors explain the dissimilarity in faunal community structure among northern MAR hydrothermal vents. 
905

906

907

908

909

910

911

912

Despite difficulties in sampling the steep edifices of RB and the higher sampling effort on LS,

this study reveals high consistencies in the taxonomic composition of species associated with mussel assemblages at the field scale. We also observed distinct patterns of abundances and diversity across the three studied vent fields, with MG appearing as a particular case. The presence of a relatively strong current at shallower depth trending southeast to northwest near MG probably acts as a strong barrier to larval dispersal with the two other vent fields.

On the other hand, the higher faunal similarity between the two geographically more distant fields ( $L S$ and RB) may be related to the presence of a current along the flank of the rift valley that may favor larval dispersal. The addition of hydrodynamic processes in future analyses may help identifying the factors driving connectivity and therefore explain compositional differences between fields. Moreover, supplementary chemical measurements to understand the speciation and availability of sulfide or metallic chemical species in the mussel habitat are needed. Particularly, we need to study the variations of the chemical species of interest - including nutrients and stressors- in the mixing zone and this, from the colonized area to the end-member fluids at the scale of a single edifice.

\section{DOI of the cruises involved}

CANNAT Mathilde, BLANDIN Jérôme, SARRADIN Pierre-Marie (2011) MOMARSAT2011 cruise, RV Pourquoi pas?, https://doi.org/10.17600/11030070

CANNAT Mathilde, SARRADIN Pierre-Marie (2012) MOMARSAT2012 cruise, RV Thalassa, https://doi.org/10.17600/12040050

LALLIER François (2013) BIOBAZ 2013 cruise, RV Pourquoi pas ?, https://doi.org/10.17600/13030030

\section{Acknowledgments}


930

931

932

933

934

935

936

937

938

939

940

941

942

943

944

945

946

947

948

949

950

951

We are grateful to the R/V Pourquoi pas? and R/V Thalassa crews for their steadfast collaboration in the success of the BIOBAZ and Momarsat cruises, the chief scientists of these cruises and the Victor6000 submersible pilots for their patience and constant support.

We are also grateful to the LEP technical and engineering teams for their valuable help both at sea and in the lab. BH was supported by the "Laboratoire d'Excellence" LabexMER (ANR10-LABX-19) and co-funded by a grant from the French government as part of the "Investissements d'Avenir" program. The project is part of the EMSO-Azores (http://www.emso-fr.org) Regional node, and of the EMSO ERIC Research Infrastructure (http://emso.eu/). The research program was funded by an ANR research grant (ANR Lucky Scales ANR-14-CE02-0008-02). The manuscript was professionally edited by Carolyn EngelGautier.

\section{References}

Barreyre, T. et al. (2012) 'Structure, temporal evolution, and heat flux estimates from the Lucky Strike deep-sea hydrothermal field derived from seafloor image mosaics', Geochemistry, Geophysics, Geosystems, 13(4), p. Q04007.

Baldrighi Elisa, Zeppilli Daniela, Crespin Rosalie, Chauvaud Pierre, Pradillon Florence, Sarrazin Jozee (2018). Colonization of synthetic sponges at the deep-sea Lucky Strike hydrothermal vent field (Mid-Atlantic Ridge): a first insight. Marine Biodiversity, 48(1), 89-103.

Bennett SA, Van Dover C, Breier JA, Coleman M. (2015) Effect of depth and vent fluid composition on the carbon sources at two neighboring deep-sea hydrothermal vent fields (Mid-Cayman Rise). Deep-Sea Research part I -Oceanographic research paper, 104: 122-133. 
952

953

954

955

956

957

958

959

960

961

962

963

964

965

966

967

968

969

970

971

Cannat M, Sarradin PM, Blandin J, Escartin, Colaco A and theMoMAR-Demo Scientific Party.

(2011). MoMar-Demo at LuckyStrike. A near-realtime multi-disciplinary observatory of hydrothermal processes and ecosystems at the Mid-Atlantic Ridge. In: AbstractOS22A-05 presented at 2011 Fall Meeting, AGU, San Francisco, CA, 5-9 Dec.

Cannat Mathilde, Lagabrielle Yves, Bougault Henri, Casey Jack, De Coutures Nathalie, Dmitriev Leonid, Fouquet Yves (1997). Ultramafic and gabbroic exposures at the Mid-Atlantic Ridge: geological mapping in the 15 degrees $\mathrm{N}$ region. Tectonophysics, 279(1-4), 193-213.

Carney, R. S. (1994). Consideration of the oasis analogy for chemosynthetic communities at Gulf of Mexico hydrocarbon vents. Geo-Marine Letters, 14(2-3), 149-159.

Carney, R. (2005) 'Zonation of Deep Biota on Continental Margins', in Gibson, R., Gordon, J., and Atkinson, R. (eds) Oceanography and Marine Biology. CRC Press, pp. 211-278.

Cerqueira, T., Pinho, D., Froufe, H., Santos, R. S., Bettencourt, R., \& Egas, C. (2017). Sediment microbial diversity of three deep-sea hydrothermal vents southwest of the Azores. Microbial ecology, 74(2), 332-349.

Charlou, J. L. et al. (2000) 'Compared geochemical signatures and the evolution of Menez Gwen $\left(37^{\circ} 50^{\prime} \mathrm{N}\right)$ and Lucky Strike $\left(37^{\circ} 17^{\prime} \mathrm{N}\right)$ hydrothermal fluids, south of the Azores Triple Junction on the Mid-Atlantic Ridge', Chemical Geology, 171(1-2), pp. 49-75.

Charlou, J. L. et al. (2002) 'Geochemistry of high $\mathrm{H}^{2}$ and $\mathrm{CH}_{4}$ vent fluids issuing from ultramafic rocks at the Rainbow hydrothermal field $\left(36^{\circ} 14^{\prime} \mathrm{N}, \mathrm{MAR}\right)^{\prime}$, Chemical Geology, 191(4), pp. 345-359. 
Chase, M. R. et al. (1998) 'Bathymetric patterns of genetic variation in a deep-sea protobranch bivalve, <Emphasis Type="Italic">Deminucula atacellana</Emphasis>', Marine

974 Biology, 131(2), pp. 301-308.

976 (2018). Sulfate minerals control dissolved rare earth element flux and $\mathrm{Nd}$ isotope signature 977 of buoyant hydrothermal plume (EMSO-Azores, $37^{\circ} \mathrm{N}$ Mid-Atlantic Ridge). Chemical 978 Geology, 499, 111-125.

979 Colaço, A., Dehairs, F. and Desbruyères, D. (2002) 'Nutritional relations of deep-sea 980 hydrothermal fields at the Mid-Atlantic Ridge: a stable isotope approach', Deep Sea 981 Research Part I: Oceanographic Research Papers, 49(2), pp. 395-412.

982 Colaço, A., Desbruyères, D., and Guezennec, J. (2007). Polar lipid fatty acids as indicators of 983 trophic associations in a deep-sea vent system community. Mar. Ecol. 28, 15-24.

984

985 986

Comtet, T. and Desbruyeres, D. (1998) 'Population structure and recruitment in mytilid bivalves from the Lucky Strike and Menez Gwen', Marine Ecology Progress Series, 163, pp. $165-177$.

Copley, J. T. P. et al. (1997) 'Spatial and interannual variation in the faunal distribution at Broken Spur vent field (29 N, Mid-Atlantic Ridge)', Marine Biology, 129(4), pp. 723-733.

Copley, J. T. P., Jorgensen, P. B. K. and Sohn, R. A. (2007) 'Assessment of decadal-scale ecological change at a deep Mid-Atlantic hydrothermal vent and reproductive time-series in the shrimp Rimicaris exoculata', Journal of the Marine Biological Association of the United Kingdom, 87(04), pp. 859-867. 
993

994

995

996

997

998

999

1000

1001

1002

1003

1004

1005

1006

1007

1008

1009

1010

1011

1012

Corbera, J.; Segonzac, M.; Cunha, M. R. (2008). A new deep-sea genus of Nannastacidae

(Crustacea, Cumacea) from the Lucky Strike hydrothermal vent field (Azores Triple Junction, Mid-Atlantic Ridge). Marine Biology Research. 4(3): 180-192.

Cordes, E. E. et al. (2007) 'Cold seeps of the deep Gulf of Mexico: Community structure and biogeographic comparisons to Atlantic equatorial belt seep communities', Deep Sea Research Part I: Oceanographic Research Papers, 54(4), pp. 637-653.

Cordes, E. E. et al. (2010) 'Influence of foundation species, depth, and location on diversity and community composition at Gulf of Mexico lower-slope cold seeps', Deep Sea Research Part II: Topical Studies in Oceanography. (Gulf of Mexico Cold Seeps), 57(21-23), pp. 18701881.

Cotte, L., V. Chavagnac, E. Pelleter, A. Laës-Huon, C. Cathalot, R. D. Riso, P.-M. Sarradin and M. Waeles. Metal partitioning after in-situ filtration at deep-sea vents of the Lucky Strike hydrothermal field (EMSO-Açores, Mid-Atlantic Ridge, $37^{\circ} \mathrm{N}$ ). In press, Deep-Sea Research Part I: Oceanographic Research Papers.

Crépeau, V., Bonavita, M.-A.C., Lesongeur, F., Randrianalivelo, H., Sarradin, P.-M., Sarrazin, J., and Godfroy, A. (2011). Diversity and function in microbial mats from the Lucky Strike hydrothermal vent field. FEMS Microbiol. Ecol. 76, 524-540.

Cuvelier, D. et al. (2009) 'Distribution and spatial variation of hydrothermal faunal assemblages at Lucky Strike (Mid-Atlantic Ridge) revealed by high-resolution video image analysis. Deep Sea Research Part I: Oceanographic Research Papers, 56(11), pp. 2026-2040. 
1013

1014

1015

1016

1017

1018

1019

1020

1021

1022

1023

1024

1025

1026

1027

1028

1029

1030

1031

1032

1033

1034

Cuvelier, D. et al. (2011a) 'Hydrothermal faunal assemblages and habitat characterisation at the Eiffel Tower edifice (Lucky Strike, Mid-Atlantic Ridge)', Marine Ecology, 32(2), pp. 243255.

Cuvelier, D., Sarrazin, J., Colaço, A., Copley, J.T., Glover, A.G., Tyler, P.A., Santos, R.S., and Desbruyères, D. (2011b). 'Community dynamics over 14 years at the Eiffel Tower hydrothermal edifice on the Mid-Atlantic Ridge'. Limnology and Oceanography 56, 16241640.

Cuvelier D, De Busserolles Fanny, Lavaud Romain, Floc'h Estelle, Fabri Marie-Claire, Sarradin Pierre-Marie, Sarrazin Jozee (2012). Biological data extraction from imagery - How far can we go? A case study from the Mid-Atlantic Ridge. Marine Environmental Research, 82, 15-27.

Cuvelier, D. et al. (2014a) 'First insights into macro- and meiofaunal colonisation patterns on paired wood/slate substrata at Atlantic deep-sea hydrothermal vents', Deep Sea Research Part I: Oceanographic Research Papers, 87, pp. 70-81.

Cuvelier D, Legendre P, Laes A, Sarradin PM, Sarrazin J (2014b) Rhythms and Community Dynamics of a Hydrothermal Tubeworm Assemblage at Main Endeavour Field - A Multidisciplinary Deep-Sea Observatory Approach. Plos One 9 (5): e96924.

De Busserolles, F. et al. (2009) 'Are spatial variations in the diets of hydrothermal fauna linked to local environmental conditions?', Deep Sea Research Part II: Topical Studies in Oceanography. (Marine Benthic Ecology and Biodiversity: A Compilation of Recent Advances in Honor of J. Frederick Grassle), 56(19-20), pp. 1649-1664.

Desbruyères, D. et al. (2000) 'A review of the distribution of hydrothermal vent communities along the northern Mid-Atlantic Ridge: dispersal vs. environmental controls', in Jones, M. B. 
1035

1036

1037

1038

1039

1040

1041

1042

1043

1044

1045

1046

1047

1048

1049

1050

1051

1052

1053

1054

1055

1056

et al. (eds) Island, Ocean and Deep-Sea Biology. Springer Netherlands (Developments in Hydrobiology, 152), pp. 201-216.

Desbruyères, D. et al. (2001) 'Variations in deep-sea hydrothermal vent communities on the Mid-Atlantic Ridge near the Azores plateau', Deep Sea Research Part I: Oceanographic Research Papers, 48(5), pp. 1325-1346.

Douville E, Charlou JL, Oelkers EH, Bienvenu P, Jove Colon CF, Donval JP, Fouquet Y, Prieur D, Appriou P. (2002). The Rainbow vent fluids $\left(36^{\circ} 14^{\prime} N, M A R\right)$ : the influence of ultramafic rocks and phase separation on trace metal content in Mid-Atlantic Ridge hydrothermal fluids. Chemical Geology 184: 37-48.

Dreyer, J. C., Knick, K. E., Flickinger, W. B., \& Van Dover, C. L. (2005). Development of macrofaunal community structure in mussel beds on the northern East Pacific Rise. Marine Ecology Progress Series, 302, 121-134.

Fabri, M.-C. et al. (2011) 'The hydrothermal vent community of a new deep-sea field, Ashadze-1, $1258^{\prime} \mathrm{N}$ on the Mid-Atlantic Ridge', Journal of the Marine Biological Association of the United Kingdom, 91(01), pp. 1-13.

Fouquet Yves, Charlou Jean-Luc, Donval Jean-Pierre, Radford-Knoery Joel, Pelle P, Ondreas Helene, Lourenco N, Segonzac Michel, Tivey Mk (1994). A detailed study of the Lucky-Strike hydrothermal site and discovery of a new hydrothermal site: " Menez-Gwen ». Preliminary results of DIVA 1 cruise (5-29 May, 1994). Inter-Ridge News, 3(2), 14-17.

Fouquet Y, Eissen JP, Ondreas H, Barriga F, Batiza R, Danyushevsky L (1998). Extensive volcaniclastic deposits at the Mid-Atlantic Ridge axis: results of deep-water basaltic explosive volcanic activity? Terra Nova, 10(5), 280-286. 
1057

1058

1059

1060

1061

1062

1063

1064

1065

1066

1067

1068

1069

1070

1071

1072

1073

1074

1075

1076

1077

1078

Galkin, S. V. and Goroslavskaya, E. I. (2010) 'Bottom fauna associated with Bathymodiolus azoricus (Mytilidae) mussel beds in the hydrothermal fields of the Mid-Atlantic Ridge', Oceanology, 50(1), pp. 51-60.

Gaudron, S. M. et al. (2010) 'Colonization of organic substrates deployed in deep-sea reducing habitats by symbiotic species and associated fauna', Marine Environmental Research, 70(1), pp. 1-12.

Gauthier, O., Sarrazin, J., \& Desbruyères, D. (2010). Measure and mis-measure of species diversity in deep-sea chemosynthetic communities. Marine Ecology Progress Series, 402, 285-302.

Gebruk, A. V. et al. (1997) 'Ecology and Biogeography of the Hydrothermal Vent Fauna of the Mid-Atlantic Ridge', in J.H.S. Blaxter, A. J. S., A. V. Gebruk, E. C. Southward and P. A. Tyler (ed.) Advances in Marine Biology. Academic Press (The Biogeography of the Oceans), pp. 93144.

Gebruk, A., \& Mironov, A. N. (2006). Biogeography of Atlantic hydrothermal vents. Ecosystems of Atlantic Hydrothermal Vents, 119-162.

Gollner, S. et al. (2010). Diversity of Meiofauna from the $9^{\circ} 50^{\prime} \mathrm{N}$ East Pacific Rise across a gradient of hydrothermal fluid emissions. PLoS ONE 5(8): e12321

Gooday, A. J. et al. (2004) 'A new monothalamous foraminiferan from 1000 to 6300m water depth in the Weddell Sea: morphological and molecular characterisation', Deep Sea Research Part II: Topical Studies in Oceanography. (ANDEEP (Antarctic benthic DEEP-sea) biodiversity: colonization history and recent community patterns: a tribute to Howard L. Sanders), 51(14), pp. 1603-1616. 
1079

1080

1081

1082

1083

1084

1085

1086

1087

1088

1089

1090

1091

1092

1093

1094

1095

1096

1097

1098

1099

Goroslavskaya, E. I., \& Galkin, S. V. (2011). Benthic fauna associated with mussel beds and shrimp swarms at hydrothermal fields on the Mid-Atlantic Ridge. Oceanology, 51(1), 69-79.

Govenar, B. and Fisher, C. R. (2007) 'Experimental evidence of habitat provision by aggregations of Riftia pachyptila at hydrothermal vents on the East Pacific Rise', Marine Ecology, 28(1), pp. 3-14.

Govenar, B. (2012). Energy Transfer through Food Webs at Hydrothermal Vents: Linking the Lithosphere to the Biosphere. Oceanography, 25 (1), pp. 246-255.

Gray, J. S. (2001). Antarctic marine benthic biodiversity in a world-wide latitudinal context. Polar Biology, 24(9), 633-641.

Husson, B., Sarradin, P. M., Zeppilli, D., \& Sarrazin, J. (2017). Picturing thermal niches and biomass of hydrothermal vent species. Deep Sea Research Part II: Topical Studies in Oceanography, 137, 6-25.

Jannasch HW (1985) The chemosynthetic support of life and the microbial diversity at deepsea hydrothermal vents. Proceedings of the Royal Society series B -Biological Sciences 225 (1240): 277-297.

Kim, S. and Hammerstrom, K. (2012) 'Hydrothermal vent community zonation along environmental gradients at the Lau back-arc spreading center', Deep Sea Research Part I: Oceanographic Research Papers, 62, pp. 10-19.

Laes-Huon A., Cathalot C., Legrand J., Tanguy V., Sarradin P.-M. (2016). Long-Term In Situ Survey of Reactive Iron Concentrations at the EMSO-Azores Observatory. leee Journal Of Oceanic Engineering, 41(4), 744-752. 
1100

1101

1102

1103

1104

1105

1106

1107

1108

1109

1110

1111

1112

1113

1114

1115

1116

1117

1118

1119

1120

Lahaye N, Gula J, Thurnherr AM, Reverdin G (2019) Deep Currents in the Rift Valley of the North Mid-Atlantic Ridge. Frontiers in Marine Science 6, article 597.

Langmuir, C. et al. (1997) 'Hydrothermal vents near a mantle hot spot: the Lucky Strike vent field at $37^{\circ} \mathrm{N}$ on the Mid-Atlantic Ridge', Earth and Planetary Science Letters, 148(1-2), pp. 69-91.

Langston, W. J., Bebianno, M. J., \& Burt, G. R. (1998). Metal handling strategies in molluscs. In Metal metabolism in aquatic environments (pp. 219-283). Springer, Boston, MA.

Lesongeur, F. et al. (2014) 'Export of deep-sea hydrothermal particles, indigenous thermophilic microorganisms and larvae to the surrounding Ocean', Cahiers De Biologie Marine, 55(4), pp. 409-420.

Leleu, T., Chavagnac, V., Cannat, M., Ceuleneer, G., Castillo, A., \& Menjot, L. (2015). Fluid Geochemistry of the Capelinhos Vent Site. A Key to Understand the Lucky Strike Hydrothermal Vent Field ( $\left.37^{\circ} \mathrm{N}, \mathrm{MAR}\right)$. In AGU Fall Meeting Abstracts.

Luther, G. W. et al. (2001) 'Chemical speciation drives hydrothermal vent ecology', Nature, 410(6830), pp. 813-816.

Luther GW, Gartman A, Yucel M, Madison AS, Moore TS, Nees HA, Nuzzio DB, Sen A, Lutz RA, Shank TM. (2012) Chemistry, Temperature, and Faunal Distributions at Diffuse-Flow Hydrothermal Vents Comparison of Two Geologically Distinct Ridge Systems. Oceanography 25 (1): 234-245.

Matabos, M., Plouviez, S., Hourdez, S., Desbruyères, D., Legendre, P., Warén, A., ... \& Thiébaut, E. (2011). Faunal changes and geographic crypticism indicate the occurrence of a 
1121 biogeographic transition zone along the southern East Pacific Rise. Journal of Biogeography, $112238(3), 575-594$.

1123 Marcon Y, Sahling H., Borowski C., dos Santos Ferreira, Thal J., Bohrmann G. 2013.

1124 Megafaunal distribution and assessment of total methane and sulfide consumption by 1125 mussel beds at Menez Gwen hydrothermal vent, based on geo-referenced photomosaics. 1126 Deep Sea Research I, 75, 93-109.

1127 Marsh, L. et al. (2012) 'Microdistribution of Faunal Assemblages at Deep-Sea Hydrothermal 1128 Vents in the Southern Ocean', PLoS ONE, 7(10), p. e48348.

1129 Marcaillou, C., Munoz, M., Vidal, O., Parra, T., \& Harfouche, M. (2011). Mineralogical 1130 evidence for $\mathrm{H} 2$ degassing during serpentinization at $300 \mathrm{C} / 300$ bar. Earth and Planetary 1131 Science Letters, 303(3-4), 281-290

1132 Martins I, Cosson RP, Riou V, Sarradin PM, Sarrazin J, Santos RS, Colaco A. (2011) 1133 Relationship between metal levels in the vent mussel Bathymodiolus azoricus and local 1134 microhabitat chemical characteristics of Eiffel Tower (Lucky Strike). Deep-Sea Research 58 1135 (3): 306-315.

1136 McClain, C. R. and Etter, R. J. (2005) 'Mid-domain models as predictors of species diversity 1137 patterns: bathymetric diversity gradients in the deep sea', Oikos, 109(3), pp. 555-566.

1138 Mironov, A. N., Gebruk, A. V., \& Moskalev, L. I. (2002). Biogeography of hydrothermal vent 1139 communities and obligate hydrothermal taxa. Biology of Hydrothermal Systems. Moscow: 1140 KMK Scientific Press Ltd, 410-455. 
1141 Nelson, D. C., Wirsen, C. O., \& Jannasch, H. W. (1989). Characterization of large, autotrophic

1142 Beggiatoa spp. abundant at hydrothermal vents of the Guaymas Basin. Appl. Environ.

1143 Microbiol., 55(11), 2909-2917.

1144 Nye, V., Copley, J. T., \& Tyler, P. A. (2013). Spatial variation in the population structure and 1145 reproductive biology of Rimicaris hybisae (Caridea: Alvinocarididae) at hydrothermal vents 1146 on the Mid-Cayman Spreading Centre. PLoS One, 8(3), e60319.

1147 Olu, K. et al. (2010) 'Biogeography and Potential Exchanges Among the Atlantic Equatorial 1148 Belt Cold-Seep Faunas', PLOS ONE, 5(8), p. e11967.

1149 Ondreas, H., Cannat, M., Fouquet, Y., Normand, A., Sarradin, P.M., and Sarrazin, J. (2009).

1150 Recent volcanic events and the distribution of hydrothermal venting at the Lucky Strike 1151 hydrothermal field, Mid-Atlantic Ridge. Geochem. Geophys. Geosystems 10, Q02006.

1152 Pester, N. J., Reeves, E. P., Rough, M. E., Ding, K., Seewald, J. S., \& Seyfried Jr, W. E. (2012). 1153 Subseafloor phase equilibria in high-temperature hydrothermal fluids of the Lucky Strike 1154 Seamount (Mid-Atlantic Ridge, 37 17' N). Geochimica et Cosmochimica Acta, 90, 303-322.

1155 Pineda, J., \& Caswell, H. (1998). Bathymetric species-diversity patterns and boundary 1156 constraints on vertical range distributions. Deep Sea Research Part II: Topical Studies in 1157 Oceanography, 45(1-3), 83-101.

1158 Plum, C. et al. (2016) 'Copepod colonization of organic and inorganic substrata at a deep-sea 1159 hydrothermal vent site on the Mid-Atlantic Ridge', Deep Sea Research Part II: Topical Studies 1160 in Oceanography. 
1161 Podowski, E. et al. (2010) 'Biotic and abiotic factors affecting distributions of megafauna in

1162 diffuse flow on andesite and basalt along the Eastern Lau Spreading Center, Tonga', Marine

1163 Ecology Progress Series, 418, pp. 25-45.

1164 Portail, M., Brandily, C., Cathalot, C., Colaço, A., Gélinas, Y., Husson, B., \& Sarrazin, J. (2018).

1165 Food-web complexity across hydrothermal vents on the Azores triple junction. Deep Sea

1166 Research Part I: Oceanographic Research Papers, 131, 101-120.

1167 Ramirez-Llodra E, Tyler PA, Baker MC, Bergstad OA, Clark MR, Escobar E, et al. (2011) Man

1168 and the Last Great Wilderness: Human Impact on the Deep Sea. PLoS ONE 6(8): e22588.

1169 R Core Team (2018). R: A language and environment for statistical computing. R Foundation

1170 for Statistical Computing, Vienna, Austria. URL https://www.R-project.org/.

1171 Rex, M. A. (1981). Community structure in the deep-sea benthos. Annual Review of Ecology

1172 and Systematics, 12(1), 331-353.

1173 Ricklefs, R. E., \& Schluter, D. (Eds.). (1993). Species diversity in ecological communities:

1174 historical and geographical perspectives (Vol. 414). Chicago: University of Chicago Press.

1175 Rybakova, E. and Galkin, S. (2015) 'Hydrothermal assemblages associated with different 1176 foundation species on the East Pacific Rise and Mid-Atlantic Ridge, with a special focus on 1177 mytilids', Marine Ecology, 36(S1), pp. 45-61.

1178 Sahling, H. et al. (2003) 'Depth-related structure and ecological significance of cold-seep 1179 communities-a case study from the Sea of Okhotsk', Deep Sea Research Part I: 1180 Oceanographic Research Papers, 50(12), pp. 1391-1409.

1181 Sarradin, P.-M. et al. (1998) 'Brief account of the chemical environment at hydrothermal vent mussel beds on the MAR', Cahiers de biologie marine, 39(3-4), pp. 253-254. 
1183

1184

1185

1186

1187

1188

1189

1190

1191

1192

1193

1194

1195

1196

1197

1198

1199

1200

1201

1202

1203

1204

Sarradin, P.-M. et al. (2009) 'Speciation of dissolved copper within an active hydrothermal edifice on the Lucky Strike vent field (MAR, $\left.37^{\circ} \mathrm{N}\right)^{\prime}$, Science of The Total Environment, 407(2), pp. 869-878.

Sarrazin, J., Robigou, V., Juniper, S., and Delaney, J. (1997). 'Biological and geological dynamics over four years on a high-temperature sulfide structure at the Juan de Fuca Ridge hydrothermal observatory'. Marine Ecology Progress Series. 153, 5-24.

Sarrazin, J. and Juniper, S. K. (1999) 'Biological characteristics of a hydrothermal edifice mosaic community', Marine Ecology Progress Series, 185, pp. 1-19.

Sarrazin Jozee, Juniper Sk, Massoth G, Legendre P (1999). Physical and chemical factors influencing species distributions on hydrothermal sulfide edifices of the Juan de Fuca Ridge, northeast Pacific. Marine Ecology Progress Series, 190, 89-112.

Sarrazin, J. et al. (2015) 'Biodiversity patterns, environmental drivers and indicator species on a high-temperature hydrothermal edifice, Mid-Atlantic Ridge', Deep Sea Research Part II: Topical Studies in Oceanography. (Exploring New Frontiers in Deep-Sea Research: In Honor and Memory of Peter A. Rona), 121, pp. 177-192.

Schlitzer, R. (2000). Electronic atlas of WOCE hydrographic and tracer data now available. Eos, Transactions American Geophysical Union, 81(5), 45-45.

Schmidt C, Le Bris N, Gaill F. (2008). Interactions of deep-sea vent invertebrates with their environment: The case of Rimicaris exoculata. Journal of Shellfish Research 27 (1): 79-90.

Sen, A. et al. (2013) 'Distribution of mega fauna on sulfide edifices on the Eastern Lau Spreading Center and Valu Fa Ridge', Deep Sea Research Part I: Oceanographic Research Papers, 72, pp. 48-60. 
Teske, A., \& Nelson, D. C. (2006). The genera Beggiatoa and Thioploca. The Prokaryotes:

Volume 6: Proteobacteria: Gamma Subclass, 784-810.

Tsurumi, M. (2003). Diversity at hydrothermal vents. Global Ecology and Biogeography, 12(3), 181-190.

Van Dover, C. L. and Doerries, M. b. (2005) 'Community structure in mussel beds at Logatchev hydrothermal vents and a comparison of macrofaunal species richness on slowand fast-spreading mid-ocean ridges', Marine Ecology, 26(2), pp. 110-120.

Van Dover, C. L. and Trask, J. L. (2000) 'Diversity at deep-sea hydrothermal vent and intertidal mussel beds', Marine Ecology Progress Series, 195, pp. 169-178.

Van Dover, C. L. (2002) 'Community structure of mussel beds at deep-sea hydrothermal vents', Marine Ecology Progress Series, 230, pp. 137-158.

Van Dover, C. L. (2003). Variation in community structure within hydrothermal vent mussel beds of the East Pacific Rise. Marine Ecology Progress Series, 253, 55-66.

Van Dover, C. L. (1995). Ecology of mid-Atlantic ridge hydrothermal vents. Geological Society, London, Special Publications, 87(1), 257-294.

1221 effluents and larvae by submesoscale and tidal currents. Deep Sea Research Part I: Oceanographic Research Papers, 133, 1-18.

1223 Vuillemin, R. et al. (2009) 'CHEMINI: A new in situ CHEmical MINlaturized analyzer', Deep 1224 Sea Research Part I: Oceanographic Research Papers, 56(8), pp. 1391-1399. 
1225 Wetzel, L. R. and Shock, E. L. (2000) ‘Distinguishing ultramafic-from basalt-hosted submarine

1226 hydrothermal systems by comparing calculated vent fluid compositions', Journal of

1227 Geophysical Research: Solid Earth, 105(B4), pp. 8319-8340.

1228 Wheeler, A. J., Murton, B., Copley, J., Lim, A., Carlsson, J., Collins, P. Benzie,, J. (2013).

1229 Moytirra: Discovery of the first known deep-sea hydrothermal vent field on the slow1230 spreading Mid-Atlantic Ridge north of the Azores. Geochemistry, Geophysics, Geosystems, 1231 14(10), 4170-4184.

1232 Wright, D., Currie, D. and Maurer, B. A. (1993) 'Energy supply and patterns of species 1233 richness on local and regional scales', Species Diversity in Ecological Communities : Historical 1234 and Geographical Perspectives, pp. 66-74.

1235 Zekely, J.; Van Dover, CL; Nemeschkal, HL; Bright, M (2006) 'Hydrothermal vent meiobenthos 1236 associated with mytilid mussel aggregations from the Mid-Atlantic Ridge and the East Pacific 1237 Rise', Deep Sea Research Part I: Oceanographic Research Papers, 53(8), pp. 1363-1378.

1238 Zeppilli, D. et al. (2015) 'Rapid colonisation by nematodes on organic and inorganic substrata 1239 deployed at the deep-sea Lucky Strike hydrothermal vent field (Mid-Atlantic Ridge)', Marine 1240 Biodiversity, 45(3), pp. 489-504. 


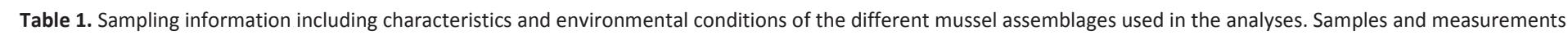

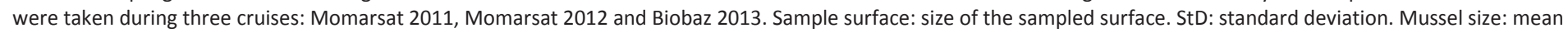

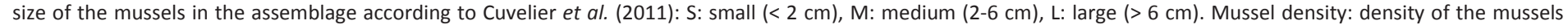

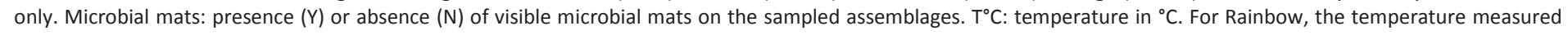
5 during dive 520 on large mussels was used to characterize the other large mussel assemblages from this field. n.a.: not available.

\begin{tabular}{|c|c|c|c|c|c|c|c|c|c|c|c|c|c|c|}
\hline \multirow[b]{2}{*}{$\begin{array}{l}\text { Vent } \\
\text { field }\end{array}$} & \multirow[b]{2}{*}{ Edifice } & \multirow[b]{2}{*}{$\begin{array}{l}\text { Sam } \\
\text { ple }\end{array}$} & \multicolumn{4}{|c|}{ Exogenous factors } & \multicolumn{8}{|l|}{ Endogenous factors } \\
\hline & & & $\begin{array}{l}\text { Depth } \\
\text { (m) }\end{array}$ & $\begin{array}{l}\quad\left({ }^{\circ} \mathrm{C}\right) \text { end- } \\
\text { member fluids }\end{array}$ & $\begin{array}{l}\text { Distance to Menez } \\
\text { Gwen }(\mathrm{km})\end{array}$ & \begin{tabular}{|l} 
Distance to \\
Montsegur (km)
\end{tabular} & $\begin{array}{l}\text { Sample surface mean } \\
\text { (st dev) } \mathrm{cm}^{2}\end{array}$ & $\begin{array}{l}\text { Mussel } \\
\text { assemblage } \\
\text { size }\end{array}$ & $\begin{array}{l}\text { Mussel density } \\
\text { (ind. } / \mathrm{m}^{2} \text { ) }\end{array}$ & $\begin{array}{l}\text { Total density of associated } \\
\text { fauna (ind. } / \mathrm{m}^{2} \text { ) }\end{array}$ & $\begin{array}{l}\text { Bacterial } \\
\text { mats }\end{array}$ & $\begin{array}{l}\text { Mean (st } \\
\text { dev) } \mathrm{T}^{\circ} \mathrm{C}\end{array}$ & \begin{tabular}{|l|} 
Max \\
$T^{\circ} \mathrm{C}$
\end{tabular} & $\begin{array}{l}\text { Min } \\
\mathrm{T}^{\circ} \mathrm{C}\end{array}$ \\
\hline \multirow{15}{*}{$\begin{array}{l}\text { Lucky } \\
\text { Strike }\end{array}$} & \multirow{3}{*}{$\begin{array}{l}\text { Cypres } \\
\text { s }\end{array}$} & CY1 & 1740 & \multirow[t]{3}{*}{304} & 99.0 & 0.7 & $228(20)$ & s & 46092 & 42762 & $\mathrm{~N}$ & $5.6(0.3)$ & 7.8 & 5.1 \\
\hline & & $\mathrm{CY} 2$ & 1739 & & 99.0 & 0.7 & $169(9)$ & $\mathrm{s}$ & 28094 & 52646 & $\mathrm{~N}$ & $5.6(0.3)$ & 7.8 & 5.1 \\
\hline & & $\mathrm{CY} 3$ & 1740 & & 99.0 & 0.7 & $318(17)$ & L & 5126 & 17581 & $\mathrm{~N}$ & $6.2(1.1)$ & 10.0 & 4.9 \\
\hline & \multirow[t]{3}{*}{$\mathrm{Y3}$} & Y31 & 1728 & \multirow[t]{3}{*}{325} & 98.6 & 0.4 & 206 (14) & $\mathrm{s}$ & 8871 & 24481 & $\mathrm{Y}$ & $6.8(0.7)$ & 8.0 & 5.6 \\
\hline & & Y32 & 1728 & & 98.6 & 0.4 & $279(43)$ & $M$ & 8131 & 23067 & $\mathrm{~N}$ & $7.9(1.2)$ & 11.8 & 4.5 \\
\hline & & Y33 & 1729 & & 98.6 & 0.4 & $169(9)$ & M & 24793 & 60508 & $\mathrm{~N}$ & $7.9(1.2)$ & 11.8 & 4.5 \\
\hline & \multirow{6}{*}{$\begin{array}{l}\text { Eiffel } \\
\text { Tower }\end{array}$} & TE1 & 1692 & \multirow[t]{6}{*}{325} & 98.5 & 0.1 & $856(52)$ & L & 2209 & 18678 & $\mathrm{~N}$ & $11.2(4.3)$ & 29.7 & 4.6 \\
\hline & & TE2 & 1697 & & 98.5 & \begin{tabular}{|l|}
0.1 \\
\end{tabular} & $122(34)$ & s & 34395 & 49427 & $\mathrm{~N}$ & $5.3(0.7)$ & 11.2 & 4.9 \\
\hline & & TE3 & 1693 & & 98.5 & 0.1 & $283(22)$ & M & 3822 & 83840 & $\mathrm{Y}$ & $5.3(0.6)$ & 8.5 & 4.5 \\
\hline & & TE4 & 1690 & & 98.5 & 0.1 & $442(106)$ & M & 7473 & 232414 & Y & $7.3(0.7)$ & 11.2 & 4.9 \\
\hline & & TE5 & 1691 & & 98.5 & 0.1 & $333(90)$ & L & 7541 & 79551 & $\mathrm{~N}$ & $11.2(4.3)$ & 29.7 & 4.6 \\
\hline & & TE6 & 1690 & & 98.5 & 0.1 & $308(13)$ & $\mathrm{s}$ & 28800 & 31657 & $\mathrm{~N}$ & $5.4(0.6)$ & 9.4 & 4.5 \\
\hline & \multirow[t]{3}{*}{$\begin{array}{l}\text { Monts } \\
\text { egur }\end{array}$} & $\begin{array}{l}\mathrm{MO} \\
1 \\
\end{array}$ & 1701 & \multirow[t]{3}{*}{316} & 98.6 & 0.0 & $175(3)$ & M & 23871 & 126437 & $\mathrm{~N}$ & $5.1(0.3)$ & 6.8 & 4.5 \\
\hline & & \begin{tabular}{|l}
$\mathrm{MO}$ \\
2
\end{tabular} & 1700 & & 98.6 & 0.0 & $373(56)$ & $\mathrm{M}$ & 4610 & 100380 & $\mathrm{~N}$ & $7.8(1.4)$ & 14.2 & 4.5 \\
\hline & & $\begin{array}{l}\mathrm{MO} \\
3\end{array}$ & 1701 & & 98.6 & 0.0 & $311(9)$ & M & 6981 & 62253 & $Y$ & $6.4(0.8)$ & 10.5 & 4.5 \\
\hline \multirow[t]{3}{*}{$\begin{array}{l}\text { Menez } \\
\text { Gwen }\end{array}$} & \multirow[t]{3}{*}{ Atos 10} & $\begin{array}{l}\text { MG } \\
1\end{array}$ & 827 & \multirow[t]{3}{*}{284} & 0.0 & 98.6 & $288(17)$ & L & 2427 & 32873 & $\mathrm{~N}$ & $9.6(0.3)$ & 18.6 & 8.8 \\
\hline & & $\begin{array}{l}M G \\
2\end{array}$ & 826 & & 0.0 & 98.6 & $431(16)$ & L & 1555 & 11487 & $\mathrm{~N}$ & $10.3(3.1)$ & 25.5 & 8.8 \\
\hline & & \begin{tabular}{|l}
$M G$ \\
3 \\
\end{tabular} & 827 & & 0.0 & 98.6 & 390 (11) & $\mathrm{L}$ & 1874 & 50675 & $\mathrm{~N}$ & $9.8(0.4)$ & 15.3 & 8.8 \\
\hline \multirow[t]{3}{*}{$\begin{array}{l}\text { Rainbo } \\
w\end{array}$} & $\begin{array}{l}\text { France } \\
5\end{array}$ & $\mathrm{Rb} 1$ & \multirow[t]{3}{*}{2274} & \multirow[t]{3}{*}{362} & \multirow[t]{3}{*}{304.6} & \multirow[t]{3}{*}{206.2} & n.a. & L & n.a. & n.a. & $\mathrm{N}$ & \multirow[t]{3}{*}{$7.8(6.1)$} & \multirow[t]{3}{*}{\begin{tabular}{|l|}
39.7 \\
\end{tabular}} & \multirow[t]{3}{*}{3.9} \\
\hline & \multirow{2}{*}{$\begin{array}{l}\text { Thermi } \\
\text { tière }\end{array}$} & $\mathrm{Rb} 2$ & & & & & $992(36)$ & L & 756 & 8002 & $\mathrm{~N}$ & & & \\
\hline & & $\mathrm{Rb} 3$ & & & & & $375(30)$ & $\mathrm{L}$ & 1574 & 187795 & $\mathrm{~N}$ & & & \\
\hline
\end{tabular}


Table 2. Correlation coefficients between the environmental variables used at the local and regional scales. $* * * P$-values $<0.05$. T_EndMember: temperature of the end-member fluids. $T^{\circ} \mathrm{C}$ : temperature in ${ }^{\circ} \mathrm{C}, \mathrm{StD}$ : standard deviation, Dist MG : distance to Menez Gwen.

\begin{tabular}{|c|c|c|c|c|c|c|c|}
\hline Variables & Depth & T_EndM & Mean_ $\mathrm{T}^{\circ} \mathrm{C}$ & Max_T ${ }^{\circ} \mathrm{C}$ & Min_ $\mathrm{T}^{\circ} \mathrm{C}$ & StD_T ${ }^{\circ} \mathrm{C}$ & Dist_MG \\
\hline T_EndM & $0.9\left(<1.10^{-5}\right)^{* * *}$ & & & & & & \\
\hline Mean_ $\mathrm{T}^{\circ} \mathrm{C}$ & -0.38 & -0.14 & & & & & \\
\hline Max_T ${ }^{\circ} \mathrm{C}$ & 0.33 & $0.56\left(7.6 .10^{-3}\right) * * *$ & $0.58\left(1.9 .10^{-3}\right)^{* * *}$ & & & & \\
\hline Min_- $T^{\circ} \mathrm{C}$ & $-0.93\left(<1.10^{-5}\right) * * *$ & $-0.79\left(<1.10^{-5}\right) * * *$ & 0.43 & -0.10 & & & \\
\hline StD_T ${ }^{\circ} \mathrm{C}$ & $0.51\left(2.0 .10^{-2}\right)^{* * *}$ & $0.69\left(5.0 .10^{-4}\right) * * *$ & $0.47\left(6.8 .10^{-3}\right) * * *$ & $0.97\left(<1.10^{-5}\right) * * *$ & -0.30 & & \\
\hline Dist_MG & $0.89\left(<1.10^{-5}\right) * * *$ & $0.93\left(<1.10^{-5}\right) * * *$ & -0.16 & $0.65\left(1.4 .10^{-3}\right) * * *$ & $-0.69\left(<1.0 .10^{-5}\right) * * *$ & $0.75\left(<1.10^{-5}\right)^{* * *}$ & \\
\hline Dist_MO & 0.21() & $0.44\left(4.6 .10^{-2}\right)$ & 0.29 & $0.82\left(<1.0 .10^{-5}\right)$ & 0.11 & $0.74\left(1.0 .10^{-4}\right)$ & $0.63\left(2.1 .10^{-3}\right)$ \\
\hline
\end{tabular}


Table 3. Macrofaunal relative densities in each sample (\%) for the three vent fields: Lucky Strike, Menez Gwen and Rainbow. Dominant taxa in each sample in bold. RB1 densities could not be estimated, so only relative abundances used for rarefaction curves and Renyi's entropy profile are reported in this table for that sample.

\begin{tabular}{|c|c|c|c|c|c|c|c|c|c|c|c|c|c|c|c|c|c|c|c|c|c|}
\hline \multirow[t]{2}{*}{ Macrofaunal taxa } & \multicolumn{15}{|c|}{ Lucky Strike } & \multicolumn{3}{|c|}{ Menez Gwen } & \multicolumn{3}{|c|}{ Rainbow } \\
\hline & $\mathrm{CY} 1$ & $\mathrm{CY} 2$ & $\mathrm{CY} 3$ & Y31 & Y32 & Y33 & TE1 & TE2 & TE3 & TE4 & TE5 & TE6 & M01 & $\mathrm{MO2}$ & $\mathrm{MO3}$ & MG1 & MG2 & MG3 & RB1 & RB2 & RB3 \\
\hline Protolira valvatoides & 26.0 & 6.0 & 1.5 & 3.4 & 0.8 & 2.0 & 0.0 & 33.4 & 1.3 & 1.0 & 0.3 & 27.9 & 26.6 & 1.5 & 5.3 & 73.8 & 56.7 & 75.8 & 0.2 & 0.0 & 2.6 \\
\hline Lurifax vitreus & 1.0 & 0.2 & 0.0 & 0.0 & 0.0 & 0.0 & 0.0 & 0.0 & 0.6 & 0.0 & 0.0 & 0.0 & 0.0 & 0.0 & 0.0 & 0.6 & 0.0 & 0.0 & 0.0 & 0.0 & 0.0 \\
\hline Lepetodrilus atlanticus & 5.7 & 3.7 & 1.5 & 0.5 & 0.3 & 3.1 & 0.2 & 28.6 & 1.6 & 2.9 & 0.3 & 5.1 & 13.2 & 0.6 & 0.7 & 23.0 & 34.0 & 22.8 & 35.6 & 1.3 & 1.3 \\
\hline Pseudorimula midatlantica & 5.1 & 4.9 & 0.7 & 0.0 & 0.3 & 2.6 & 1.2 & 1.0 & 0.0 & 1.9 & 5.5 & 2.1 & 1.4 & 19.4 & 5.5 & 0.0 & 0.0 & 0.0 & 0.0 & 0.4 & 2.6 \\
\hline Peltospira smaragdina & 0.0 & 0.4 & 0.2 & 0.0 & 0.0 & 0.0 & 0.0 & 0.0 & 0.0 & \begin{tabular}{|l|}
0.0 \\
\end{tabular} & 0.3 & 0.0 & 0.2 & 0.2 & 0.0 & 0.0 & 0.0 & 0.0 & 0.0 & 0.0 & 0.0 \\
\hline Laeviphitus desbruyeresi & 0.0 & 0.0 & 0.0 & 0.0 & 0.0 & 1.0 & 0.0 & 0.2 & 2.2 & \begin{tabular}{|l|l|}
0.4 \\
\end{tabular} & 0.0 & 0.6 & 0.0 & 0.0 & 0.0 & 0.0 & 0.0 & 0.0 & 0.0 & 0.0 & 0.0 \\
\hline Shinkailepas briandi & 0.0 & 0.0 & 0.0 & 0.0 & 7.6 & 0.0 & 0.0 & 0.0 & 0.0 & 0.0 & 0.0 & 0.0 & 0.0 & 0.0 & 0.2 & 0.0 & 0.0 & 0.0 & 0.0 & 0.0 & 0.0 \\
\hline Lirapex costellata & 0.0 & 0.0 & 0.0 & 0.0 & 0.0 & 0.0 & 0.0 & 0.0 & 0.0 & 0.0 & 0.0 & 0.2 & 0.7 & 0.0 & 0.0 & 0.0 & 0.0 & 0.0 & 0.0 & 0.0 & 0.0 \\
\hline Xylodiscula analoga & 0.0 & 0.0 & 0.0 & 0.0 & 0.0 & 0.0 & 0.0 & 0.0 & 0.0 & 0.0 & 0.0 & \begin{tabular}{|l|l|l|}
0.1 & &
\end{tabular} & 0.0 & 0.0 & 0.2 & 0.0 & 0.0 & 0.0 & 0.0 & 0.0 & 0.0 \\
\hline Amathys lutzi & 10.8 & 25.5 & 52.0 & 0.2 & 0.8 & 15.3 & 2.2 & 11.9 & 8.1 & 28.6 & 16.0 & \begin{tabular}{|l|l|}
18.1 &
\end{tabular} & 23.3 & 24.4 & 1.2 & 0.0 & 0.0 & 0.0 & 38.1 & 78.1 & 87.2 \\
\hline Archinome & 0.0 & 0.0 & 0.0 & 0.0 & 0.0 & 0.0 & 0.0 & 0.0 & 0.0 & 0.0 & 0.0 & 0.0 & 0.0 & 0.0 & 0.0 & 0.0 & 0.0 & 0.0 & 0.3 & 0.0 & 0.0 \\
\hline Branchipolynoe seepensis & 47.4 & 53.5 & 24.4 & 11.3 & 17.4 & 50.0 & 18.8 & 20.9 & 20.9 & 43.9 & 45.5 & \begin{tabular}{|l|}
37.3 \\
\end{tabular} & 17.7 & 25.2 & 36.0 & 0.0 & 0.0 & 0.0 & 0.8 & 0.9 & 0.0 \\
\hline Branchinotogluma sp & 0.0 & 0.0 & 1.7 & 0.0 & 0.0 & 0.5 & 0.0 & 0.4 & 0.9 & 1.3 & 5.8 & 0.0 & 0.2 & 7.6 & 1.2 & 0.0 & 0.0 & 0.0 & 0.0 & 0.0 & 0.0 \\
\hline Lepidonotopodium jouinae & 0.0 & 0.0 & 3.5 & 0.0 & 0.0 & 3.1 & 0.8 & 0.0 & 0.0 & 0.2 & 1.0 & 0.0 & 0.0 & 1.3 & 0.0 & 0.0 & 0.0 & 0.0 & 0.6 & 0.4 & 1.3 \\
\hline Levensteiniella iris & 0.0 & 0.0 & 0.0 & 0.0 & 0.0 & 0.0 & 0.2 & 0.0 & 0.0 & 0.2 & 0.0 & 0.0 & 0.0 & 0.0 & 0.0 & 0.0 & 0.0 & 0.0 & 0.2 & 0.4 & 0.0 \\
\hline Glyceridae & 0.2 & 0.2 & 0.0 & 0.0 & 0.0 & 0.0 & 0.0 & 0.0 & 0.0 & 0.0 & 0.0 & 0.2 & 0.3 & 0.0 & 0.0 & 0.0 & 0.0 & 0.0 & 0.0 & 0.0 & 0.0 \\
\hline Spionidae & 0.4 & 0.4 & 0.5 & 0.0 & 0.0 & 0.5 & 0.0 & 0.4 & 0.0 & 0.2 & 0.3 & 0.0 & 2.4 & 0.4 & 0.2 & 0.0 & 0.0 & 0.0 & 2.9 & 4.8 & 3.8 \\
\hline Cirratulidae & 0.0 & 0.0 & 0.0 & 0.0 & 0.0 & 0.0 & 0.0 & 0.2 & 0.0 & \begin{tabular}{|l|}
0.0 \\
\end{tabular} & 0.0 & 0.0 & 0.2 & 0.0 & 0.0 & 0.0 & 0.0 & 0.0 & 0.0 & 0.0 & 0.0 \\
\hline Dorvilleidae & 0.0 & 0.0 & 0.0 & 0.0 & 0.0 & 0.0 & \begin{tabular}{|l|}
0.0 \\
\end{tabular} & 0.6 & 59.4 & \begin{tabular}{|l|}
8.0 \\
\end{tabular} & 5.3 & \begin{tabular}{|l|l|}
0.4 & \\
\end{tabular} & 6.9 & 13.7 & 32.1 & 0.0 & \begin{tabular}{|l|}
0.0 \\
\end{tabular} & 0.0 & 0.0 & 0.0 & 0.0 \\
\hline Hesionidae & 0.4 & 0.0 & 0.0 & 0.0 & 0.0 & 0.0 & \begin{tabular}{|l|}
0.0 \\
\end{tabular} & 0.0 & 0.0 & \begin{tabular}{|l|}
0.0 \\
\end{tabular} & 0.0 & \begin{tabular}{|l|}
0.0 \\
\end{tabular} & 0.0 & 0.0 & 0.0 & 0.0 & \begin{tabular}{|l|}
0.0 \\
\end{tabular} & 0.0 & 0.3 & 0.0 & 0.0 \\
\hline Aplacophora & 1.2 & 0.0 & 0.0 & 0.0 & 0.0 & 0.0 & 0.2 & 0.0 & 4.7 & \begin{tabular}{|l|}
0.0 \\
\end{tabular} & 0.3 & 0.2 & 1.4 & 1.9 & 11.3 & 0.3 & 0.0 & 0.0 & 0.0 & 0.0 & 0.0 \\
\hline Amphipoda & 1.2 & 0.9 & 0.2 & 72.5 & 66.4 & 5.6 & \begin{tabular}{|l|l|}
46.8 & \\
\end{tabular} & 0.8 & 0.0 & \begin{tabular}{|l|}
0.0 \\
\end{tabular} & 0.0 & \begin{tabular}{|l|l|}
1.7 &
\end{tabular} & 1.5 & 1.1 & 4.3 & 1.9 & \begin{tabular}{|l|l|}
1.4 & \\
\end{tabular} & 1.0 & 0.0 & 0.4 & 0.0 \\
\hline Euphausiidae sp. & 0.0 & 0.0 & 0.0 & 0.0 & 0.0 & 0.0 & \begin{tabular}{|l|}
0.0 \\
\end{tabular} & 0.0 & 0.0 & \begin{tabular}{|l|}
0.0 \\
\end{tabular} & 0.0 & \begin{tabular}{|l|}
0.0 \\
\end{tabular} & 0.0 & 0.0 & 0.0 & 0.0 & \begin{tabular}{|l|}
0.0 \\
\end{tabular} & 0.2 & 0.0 & 0.0 & 0.0 \\
\hline Isopoda & 0.0 & 0.0 & 0.0 & 0.0 & 0.0 & 0.0 & \begin{tabular}{|l|}
0.0 \\
\end{tabular} & 0.0 & 0.0 & \begin{tabular}{|l|}
0.0 \\
\end{tabular} & 0.0 & \begin{tabular}{|l|}
0.0 \\
\end{tabular} & 0.0 & 0.0 & 0.0 & 0.0 & \begin{tabular}{|l|l|}
7.4 & \\
\end{tabular} & 0.0 & 0.0 & 0.0 & 0.0 \\
\hline
\end{tabular}




\begin{tabular}{|l|l|l|l|l|l|l|l|l|l|l|l|l|l|l|l|l|l|l|l|l|l|l|}
\hline Segonzacia mesatlantica & 0.2 & 0.2 & 0.2 & 0.0 & 0.0 & 0.0 & 0.2 & 0.0 & 0.0 & 0.0 & 0.3 & 0.0 & 0.0 & 0.4 & 0.2 & 0.0 & 0.0 & 0.0 & 0.0 & 0.0 & 0.0 & \\
\hline Alvinocaris markensis & 0.0 & 0.0 & 0.0 & 0.0 & 0.0 & 0.0 & 0.0 & 0.0 & 0.0 & 0.0 & 0.0 & 0.0 & 0.0 & 0.0 & 0.0 & 0.0 & 0.0 & 0.0 & 0.6 & 0.0 & 0.0 \\
\hline Mirocaris fortunata & 0.6 & 4.2 & 13.4 & 12.0 & 6.1 & 16.3 & 29.4 & 0.2 & 0.3 & 5.0 & 9.8 & 1.7 & 2.6 & 2.4 & 1.0 & 0.3 & 0.5 & 0.2 & 15.7 & 7.9 & 1.3 \\
\hline Rimicaris exoculata & 0.0 & 0.0 & 0.0 & 0.0 & 0.0 & 0.0 & 0.0 & 0.0 & 0.0 & 0.0 & 0.0 & 0.0 & 0.0 & 0.0 & 0.0 & 0.0 & 0.0 & 0.0 & 0.0 & 0.4 & 0.0 & 0.0 \\
\hline Rimicaris chacei & 0.0 & 0.0 & 0.0 & 0.0 & 0.0 & 0.0 & 0.0 & 0.0 & 0.0 & 0.0 & 0.0 & 0.0 & 0.0 & 0.0 & 0.0 & 0.0 & 0.0 & 0.0 & 0.3 & 0.0 & 0.0 & \\
\hline Sericosura heteroscela & 0.0 & 0.0 & 0.0 & 0.0 & 0.5 & 0.0 & 0.0 & 1.3 & 0.0 & 0.2 & 0.0 & 0.1 & 1.0 & 0.0 & 0.2 & 0.0 & 0.0 & 0.0 & 0.3 & 0.0 & 0.0 \\
\hline Sipuncula & 0.0 & 0.0 & 0.0 & 0.0 & 0.0 & 0.0 & 0.0 & 0.2 & 0.0 & 0.0 & 0.0 & 0.0 & 0.0 & 0.0 & 0.0 & 0.0 & 0.0 & 0.0 & 0.0 & 0.0 & 0.0 \\
\hline Nemertea & 0.0 & 0.0 & 0.0 & 0.0 & 0.0 & 0.0 & 0.0 & 0.0 & 0.0 & 6.3 & 9.8 & 4.1 & 0.5 & 0.0 & 0.2 & 0.0 & 0.0 & 0.0 & 1.3 & 4.8 & 0.0 & 0.0 \\
\hline Ophiura & 0.0 & 0.0 & 0.0 & 0.0 & 0.0 & 0.0 & 0.0 & 0.0 & 0.0 & 0.0 & 0.0 & 0.0 & 0.0 & 0.0 & 0.0 & 0.0 & 0.0 & 0.0 & 2.6 & 0.0 & 0.0 \\
\hline
\end{tabular}


Table 4. Meiofaunal relative densities in each sample (\%) for the three vent fields: Lucky Strike, Menez Gwen and Rainbow. Dominant meiofauna in each sample in bold. NA: not available. RB1 densities could not be estimated, so only relative abundances used for rarefaction curves and Renyi's entropy profile are reported in this table for that sample. No meiofauna were sampled on RB3 due to technical problems with the suction sampler device.

\begin{tabular}{|c|c|c|c|c|c|c|c|c|c|c|c|c|c|c|c|c|c|c|c|c|}
\hline Meiofaunal taxa & \multicolumn{15}{|c|}{ Lucky Strike } & \multicolumn{3}{|c|}{ Menez Gwen } & \multicolumn{2}{|c|}{ Rainbow } \\
\hline & CY1 & $\mathrm{CY} 2$ & CY3 & Y31 & Y32 & Y33 & TE1 & TE2 & TE3 & TE4 & TE5 & TE6 & MO1 & MO2 & MO3 & MG1 & MG2 & MG3 & RB1 & RB2 \\
\hline Copepoda & 92.9 & 92.0 & 66.7 & 86.7 & 37.6 & 62.1 & 97.2 & 29.2 & 32.2 & 33.4 & 34.0 & 48.7 & 36.4 & 34.9 & 30.6 & 86.1 & 83.1 & 38.9 & 37.1 & 0.6 \\
\hline Nauplii & 3.5 & 0.7 & 0.0 & 0.0 & 0.0 & 0.3 & 0.5 & 0.0 & 0.0 & 1.2 & 0.1 & 3.4 & 0.1 & 0.1 & 1.0 & 11.4 & 12.2 & 1.9 & 0.0 & 0.0 \\
\hline Ostracoda & 0.0 & 0.0 & 14.8 & 8.3 & 2.8 & 26.2 & 1.8 & 0.0 & 33.8 & 7.3 & 33.7 & 20.5 & 1.0 & 32.5 & 1.3 & 0.0 & 0.0 & 0.0 & 0.0 & 0.0 \\
\hline Thalycrocuma sarradini & 0.0 & 0.0 & 0.0 & 0.0 & 0.0 & 0.0 & 0.0 & 0.0 & 0.0 & 0.0 & 0.0 & 0.0 & 0.0 & 0.0 & $<0.1$ & 0.0 & 0.0 & 0.0 & 0.0 & 0.0 \\
\hline Nematoda & 1.8 & 7.3 & 18.2 & 5.0 & 59.6 & 10.1 & 0.5 & 39.3 & 32.2 & 58.0 & 30.9 & 18.0 & 55.8 & 32.4 & 61.2 & 0.0 & 0.0 & 0.0 & 62.4 & 99.4 \\
\hline Turbellaria & 1.8 & 0.0 & 0.2 & 0.0 & 0.0 & 1.4 & 0.0 & 0.0 & 0.4 & 0.2 & 0.1 & 5.5 & 1.8 & 0.0 & 2.8 & 0.1 & 0.3 & 0.1 & 0.0 & 0.0 \\
\hline Tanaidacea & 0.0 & 0.0 & 0.0 & 0.0 & 0.0 & 0.0 & 0.0 & 28.4 & 0.1 & 0.0 & 0.0 & 1.3 & 4.7 & 0.0 & 0.0 & 0.0 & 0.0 & 0.1 & 0.0 & 0.0 \\
\hline Foraminifera & 0.0 & 0.0 & 0.0 & 0.0 & 0.0 & 0.0 & 0.0 & 1.4 & 1.2 & 0.0 & 1.1 & 0.0 & 0.1 & 0.0 & 3.0 & 2.3 & 4.3 & 59.1 & 0.6 & 0.0 \\
\hline Demospongiae & 0.0 & 0.0 & 0.0 & 0.0 & 0.0 & 0.0 & 0.0 & 1.0 & 0.1 & 0.0 & 0.0 & 0.0 & 0.0 & 0.0 & 0.0 & 0.0 & 0.0 & 0.0 & 0.0 & 0.0 \\
\hline Halacaridae & 0.0 & 0.0 & 0.0 & 0.0 & 0.0 & 0.0 & 0.0 & 0.6 & 0.0 & $<0.1$ & 0.0 & 2.7 & 0.1 & 0.0 & 0.0 & 0.0 & 0.0 & 0.0 & 0.0 & 0.0 \\
\hline
\end{tabular}


Table S1. Mean environmental conditions of (A) end-member fluids, (B) mussel assemblage habitats and (C) bottom temperatures in the Menez Gwen, Lucky Strike and Rainbow vent fields. (A) Adapted from Charlou et al. (2000, 2002) and Douville et al. (2002), (B) $\mathrm{CH}_{4}$ and Fe analyses were done during the Biobaz cruise in 2013: MG (dive 515), LS (dive 518), RB (dive 520), $\mathrm{H}_{2} \mathrm{~S}$ values were measured directly in various mussel assemblages during the Momarsat cruises (2011 \& 2012) on the Lucky Strike vent field and on MG during the Biobaz cruise (2013). Other $\mathrm{H}_{2} \mathrm{~S}$ values were extrapolated, see table footnote for details; (C) Measured during Biobaz 2013. High values are highlighted in bold.

\begin{tabular}{|c|c|c|c|}
\hline Site & Menez Gwen & Lucky Strike & Rainbow \\
\hline \multicolumn{4}{|l|}{ (A) End-member fluids } \\
\hline $\mathrm{T}\left({ }^{\circ} \mathrm{C}\right)$ & $265-284$ & $304-325$ & $360-365$ \\
\hline pH & $4.2-4.8$ & $3.5-4.9$ & $2.8-3.1$ \\
\hline $\mathrm{CH}_{4}(\mathrm{mM})$ & $1.4-2.2$ & $0.3-0.9$ & $2.2-2.5$ \\
\hline $\mathrm{Fe}(\mathrm{mM})$ & $0.02-0.03$ & $0.03-0.86$ & 24 \\
\hline $\mathrm{H}_{2} \mathrm{~S}(\mathrm{mM})$ & $1.3-1.8$ & $0.6-3.3$ & $1-2.5$ \\
\hline \multicolumn{4}{|l|}{ (B) Mussel assemblages } \\
\hline$\left[\mathrm{CH}_{4}\right](\mu \mathrm{M})$ & 0.50 & 1.31 & 36.57 \\
\hline$[\mathrm{Fe}](\mu \mathrm{M})$ measured & 0.82 & 0.25 & 3.01 \\
\hline $\mathrm{H}_{2} \mathrm{~S}(\mu \mathrm{M})$ measured in situ in the mussel assemblages & $33-51$ & $0.99-9.96$ & \\
\hline Range of $\mathrm{H}_{2} \mathrm{~S}$ available extrapolated from mean $\mathrm{T}^{\circ} \mathrm{C}$ in the mussel assemblages $(\mu \mathrm{M}) *$ & $4.6-8.6$ & 5.2-43.2 & 20.9 \\
\hline Range of $\mathrm{H}_{2} \mathrm{~S}$ available extrapolated from max $\mathrm{T}^{\circ} \mathrm{C}$ in the mussel assemblages $(\mu \mathrm{M}) *$ & $37.2-95.5$ & $14.8-162.3$ & 176.2 \\
\hline (C) Bottom $\mathrm{T}^{\circ} \mathrm{C}$ & 8.8 & 4.5 & 3.5 \\
\hline
\end{tabular}

*The range of $\mathrm{H}_{2} \mathrm{~S}$ available in mussel assemblage was estimated based on average end-member $\mathrm{H}_{2} \mathrm{~S}$ concentration of each vent field extrapolated through simple conservative mixing with background seawater. The conservative tracer used for dilution was the mean and maximum temperatures. These estimated concentrations represent "absolute" maximum concentrations available for the fauna and have to be used cautiously. Indeed, as $\mathrm{H}_{2} \mathrm{~S}$ is very reactive, it is consumed during mixing with seawater through polysulfide precipitation processes. In addition, due to conductive cooling processes in the sub-seafloor, $\mathrm{H}_{2} \mathrm{~S}$ concentrations in the fluids around the fauna may be lower compared to more focused and direct fluid outputs. 

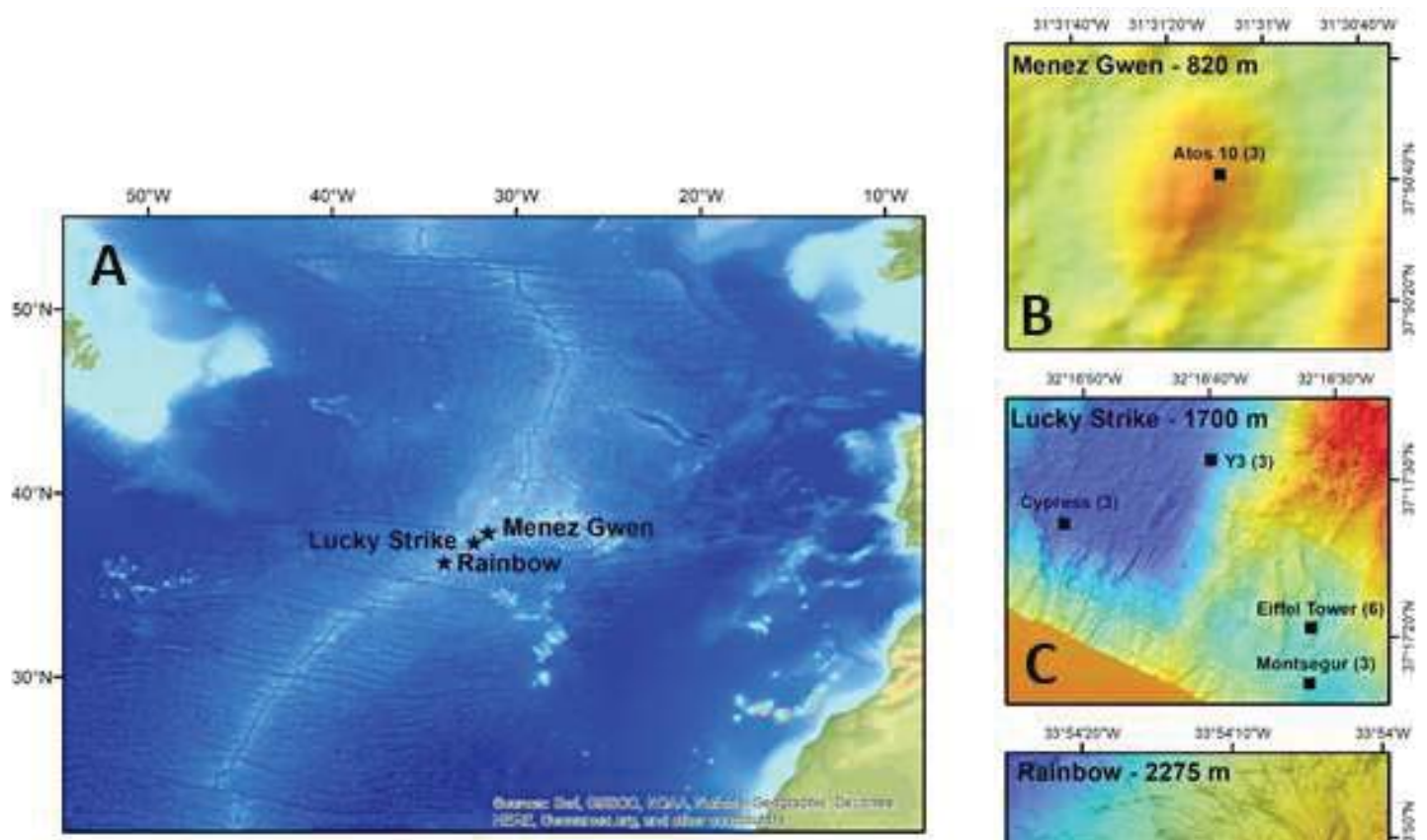

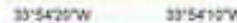

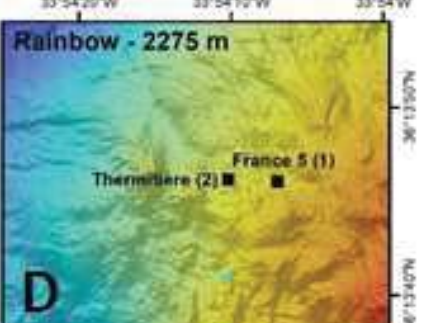

Figure 1. A. Location of the three vent fields on the Mid-Atlantic Ridge, south of the Azores: Menez Gwen at $~ 825 \mathrm{~m}$, Lucky Strike at $\sim 1700 \mathrm{~m}$ and Rainbow at $\sim 2275 \mathrm{~m}$ depth. A total of 21 mussel assemblages were sampled on seven active edifices on B. Menez Gwen, C. Lucky Strike and D. Rainbow. Number of samples in parentheses. 


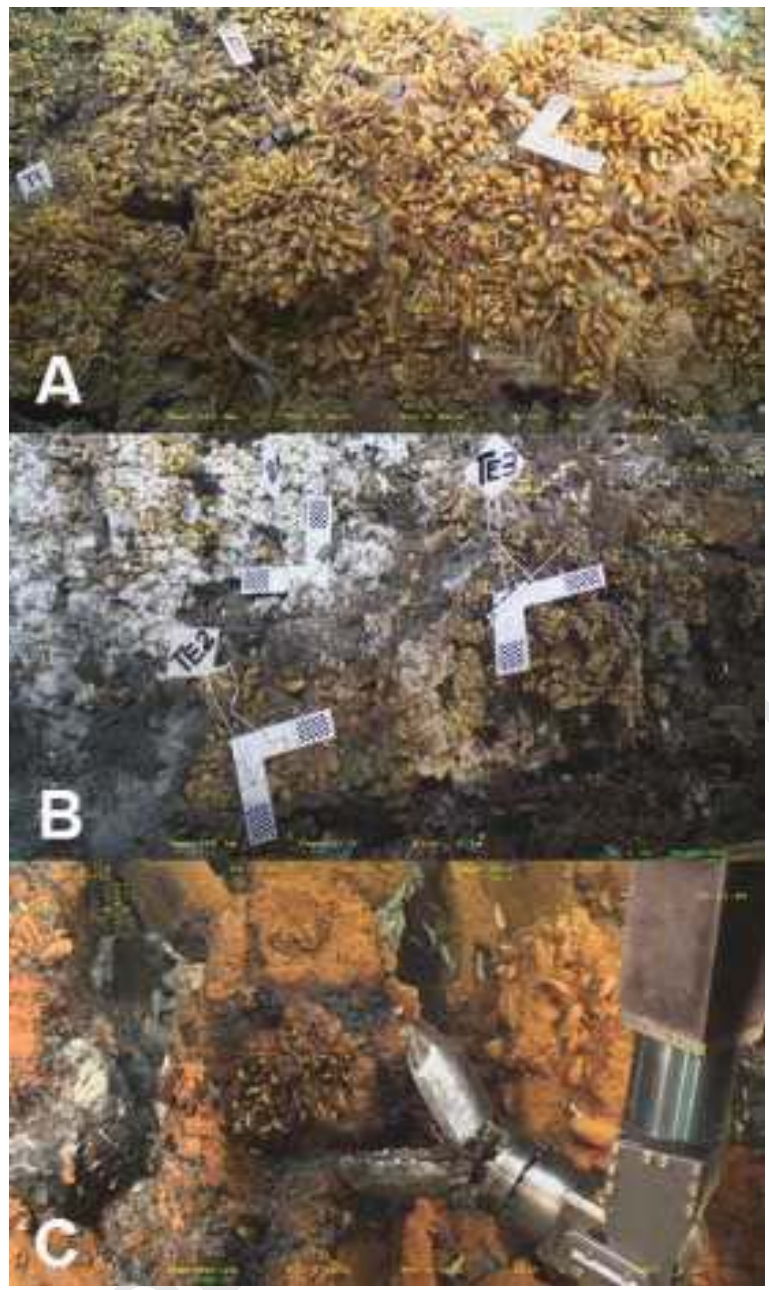

Figure 2. A. Sampling locations on the Menez Gwen (MG) vent field, at the Atos 10 site. Three mussel assemblages were characterized and sampled. Autonomous temperature probes recorded temperatures for 13 days within each assemblage. When possible, calibrated targets, with visible $7 \mathrm{x}$ $7 \mathrm{~mm}$ checkerboards, were deployed to facilitate image calibration. B. Three sampled mussel assemblages on the Lucky Strike (LS) vent field, here on the Eiffel Tower edifice in 2013. A total of 15 mussel assemblages were characterized and sampled on four different edifices (Eiffel Tower but also Y3, Cypress and Montségur) during three cruises in 2011, 2012 and 2013. Autonomous temperature probes recorded temperatures for several days within each assemblage. C. One of the three sampled mussel assemblage on the Rainbow (RB) vent field, here on the Thermitière edifice. In the photo, the Victor6000 ROV arm is being used, after a first suction sampling, to sample the mussels and associated fauna. A second suction sample is taken to clear the surface of any remaining fauna. Since no temperature probe was deployed at this site, mean temperatures were extracted from in situ measurements taken using the ROV probe. 


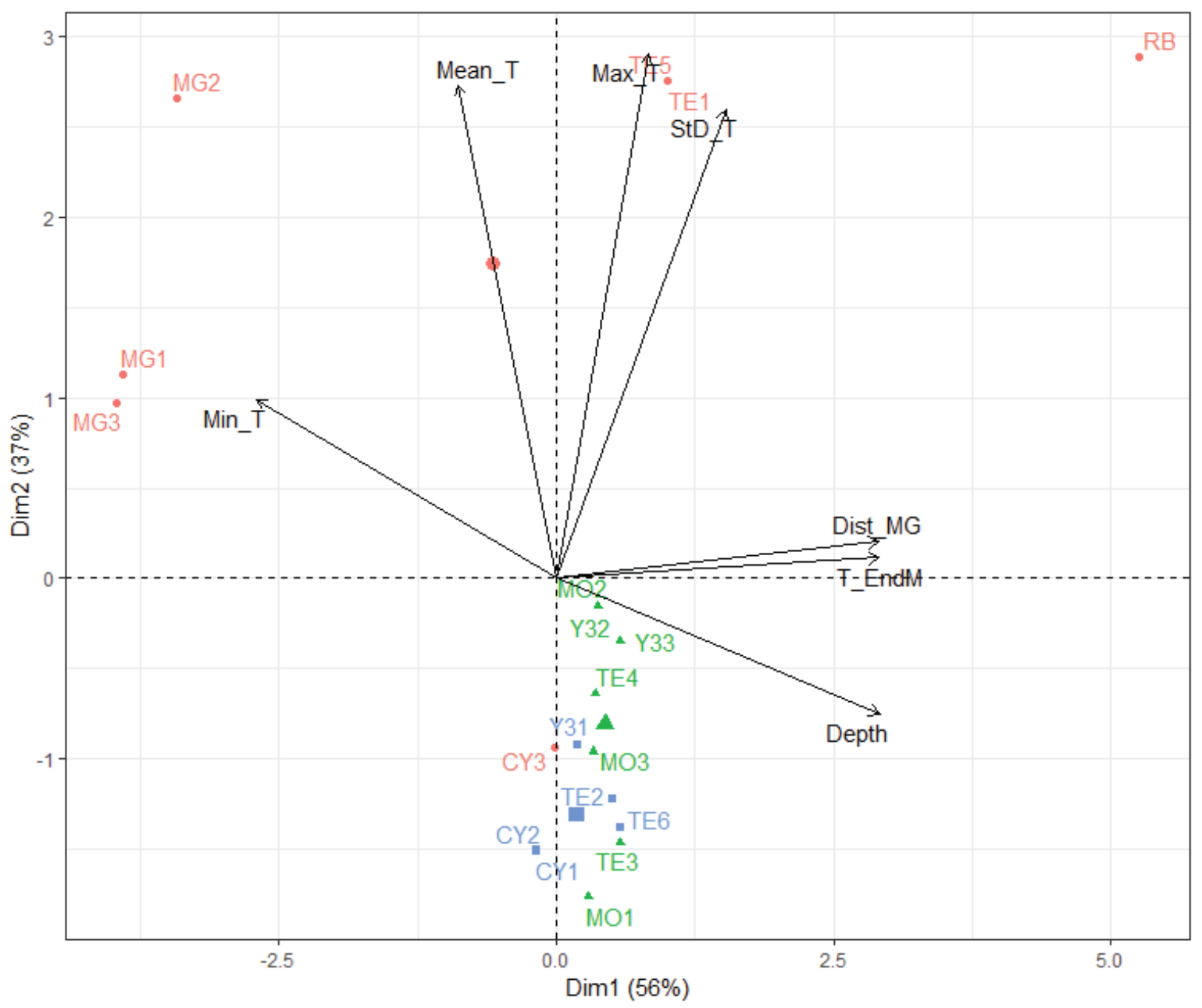

Figure 3. Biplot of the principal component analysis (PCA) of environmental variables at the local and regional scales. Colors indicate size of the mussels in the assemblages: Red: Large (> $6 \mathrm{~cm})$; Green: medium $(2-6 \mathrm{~cm})$; Blue: small mussels $(<2 \mathrm{~cm})$. Environmental variables are the following: minimum temperature (Min_T), mean temperature (Mean_T), maximum temperature (Max_T), standard deviation of temperature (Std_T), distance to Menez Gwen in kilometres (Dist_MG), temperature of end-member fluids $-T$-EndM) and depth. 

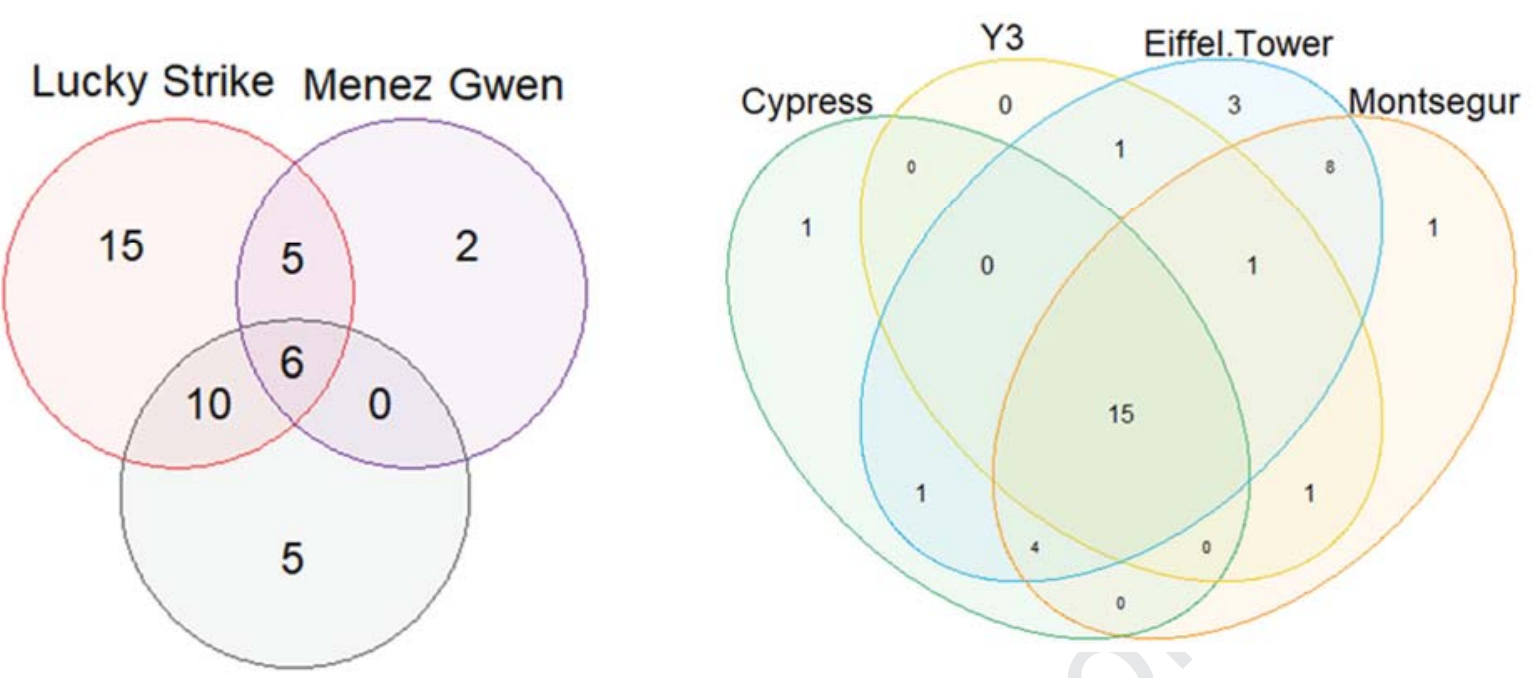

Rainbow

Figure 4. Venn diagrams showing distribution of meio- and macrofaunal taxa. A. among the three vent fields and B. among four edifices of the Lucky Strike vent field and for two years on Eiffel Tower. 


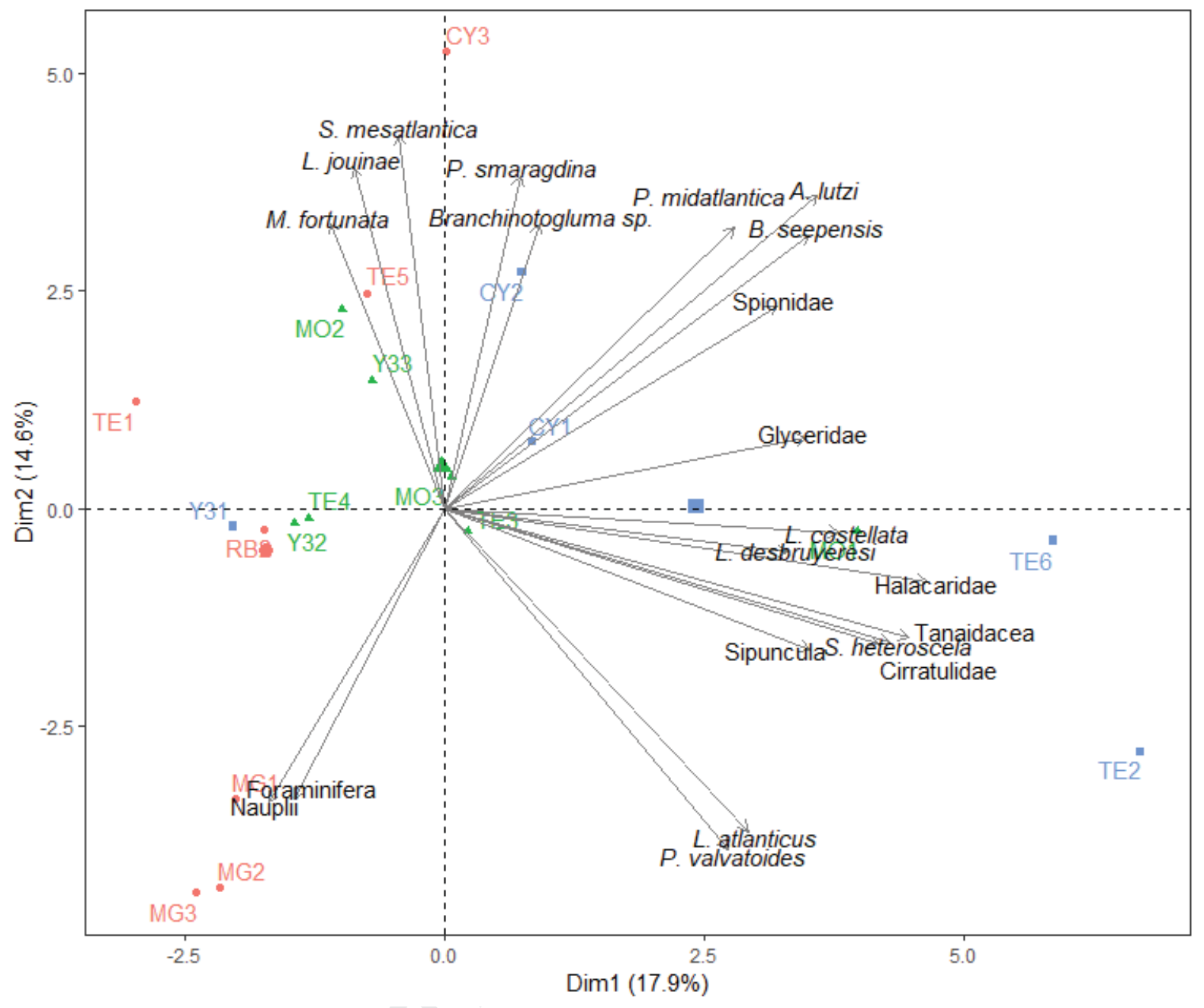

Figure 5. Principal component analysis (PCA) of species composition on the different edifices sampled in this study. Variables (taxa) not well represented ( $\cos 2<25 \%$ ) are not shown. Colors and shapes distinguish the different assemblage types: blue squares: small mussels $(<$ $2 \mathrm{~cm}$ ); green triangles: medium mussels $(2-6 \mathrm{~cm})$; red circles: large mussels $(>6 \mathrm{~cm})$. Larger symbols indicate the barycenter of assemblage type. 


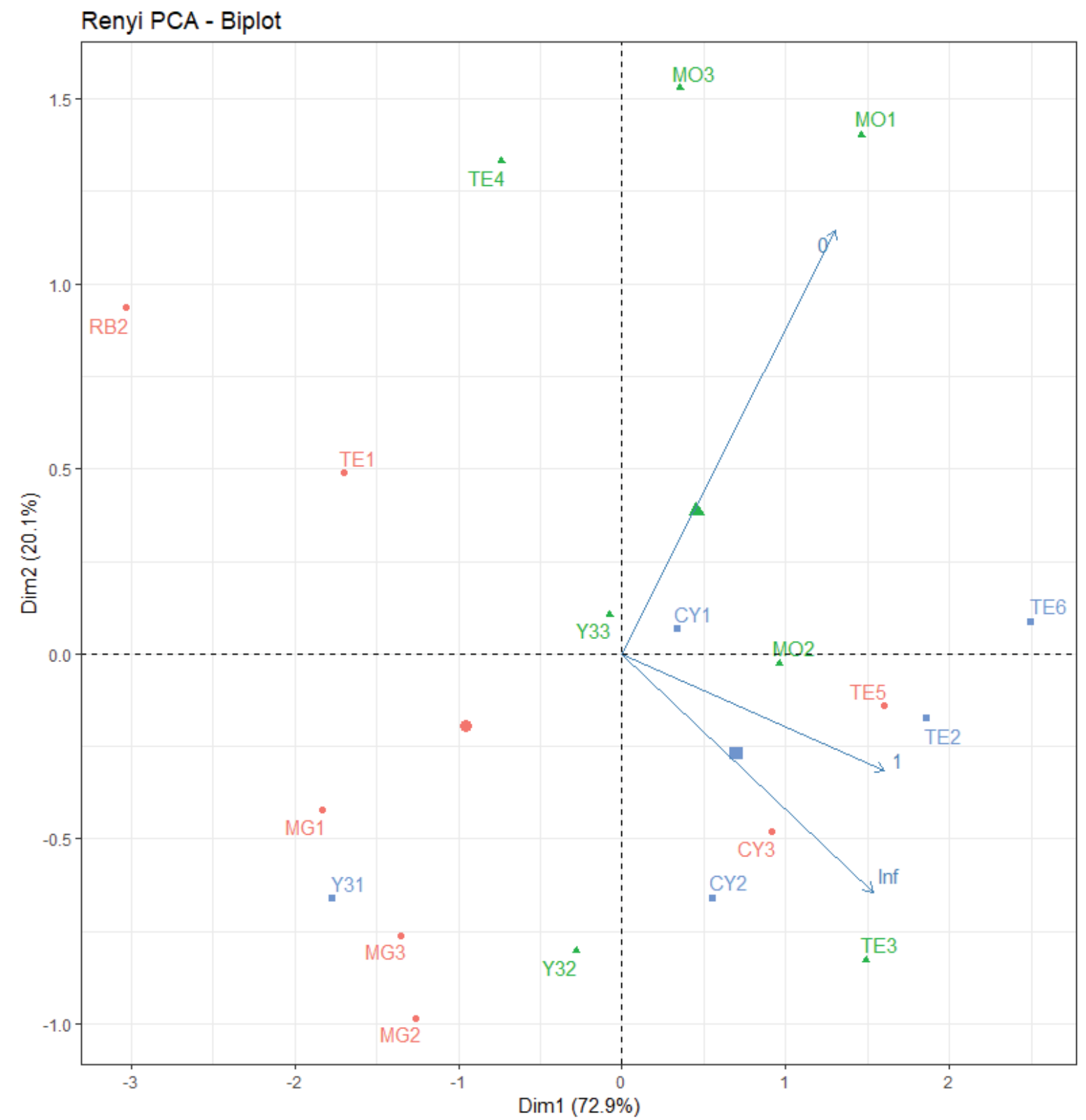

Figure 6. Principal component analysis on Renyi's entropy selected values. Colors indicate the type of assemblage: Red: Large $(>6 \mathrm{~cm})$; Green: medium $(2-6 \mathrm{~cm})$; Blue: small mussels $(<2 \mathrm{~cm})$. 


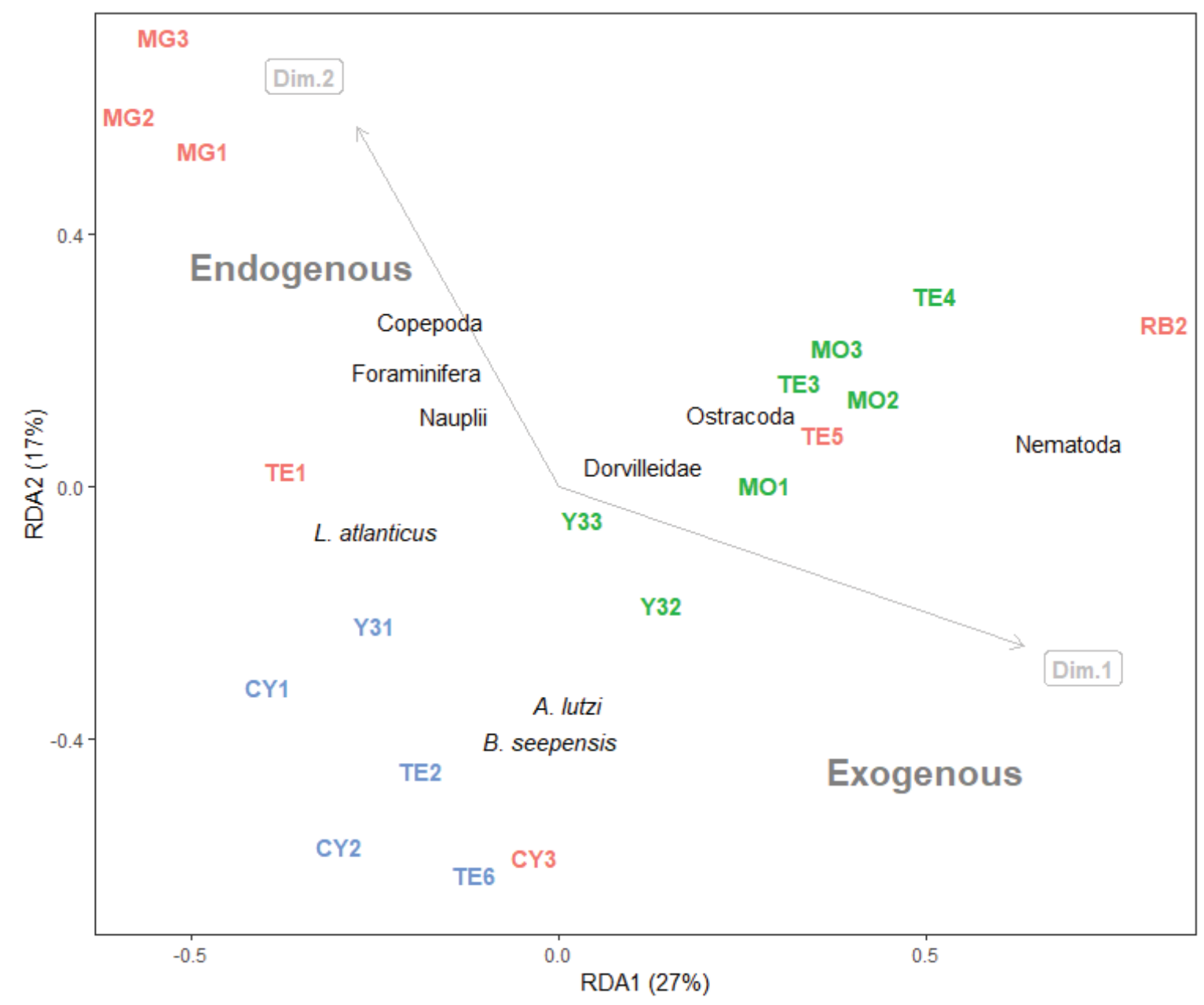

Figure 7. Redundancy analysis of taxonomic composition explained by environmental variables. Dim 1 and 2 represent the position of the two first dimensions of the PCA on environmental variables (Figure 3). Dim. 1 was highly correlated with exogenous factors: Depth, end-member temperature, distance to the Menez Gwen vent field. Dim. 2 was highly correlated to endogenous factors: Min, Mean, Max and Std temperatures taken within the mussel assemblages. Colors indicate the type of assemblage: Red: Large (> 6 $\mathrm{cm})$; Green: medium $(2-6 \mathrm{~cm})$; Blue: small mussels $(<2 \mathrm{~cm})$. Only species with coordinates distinct from the center (absolute value $>0.1$ ) are shown. The plot is adapted from the default output of rda function from "vegan" package using scaling=2 (correlation triplot). 


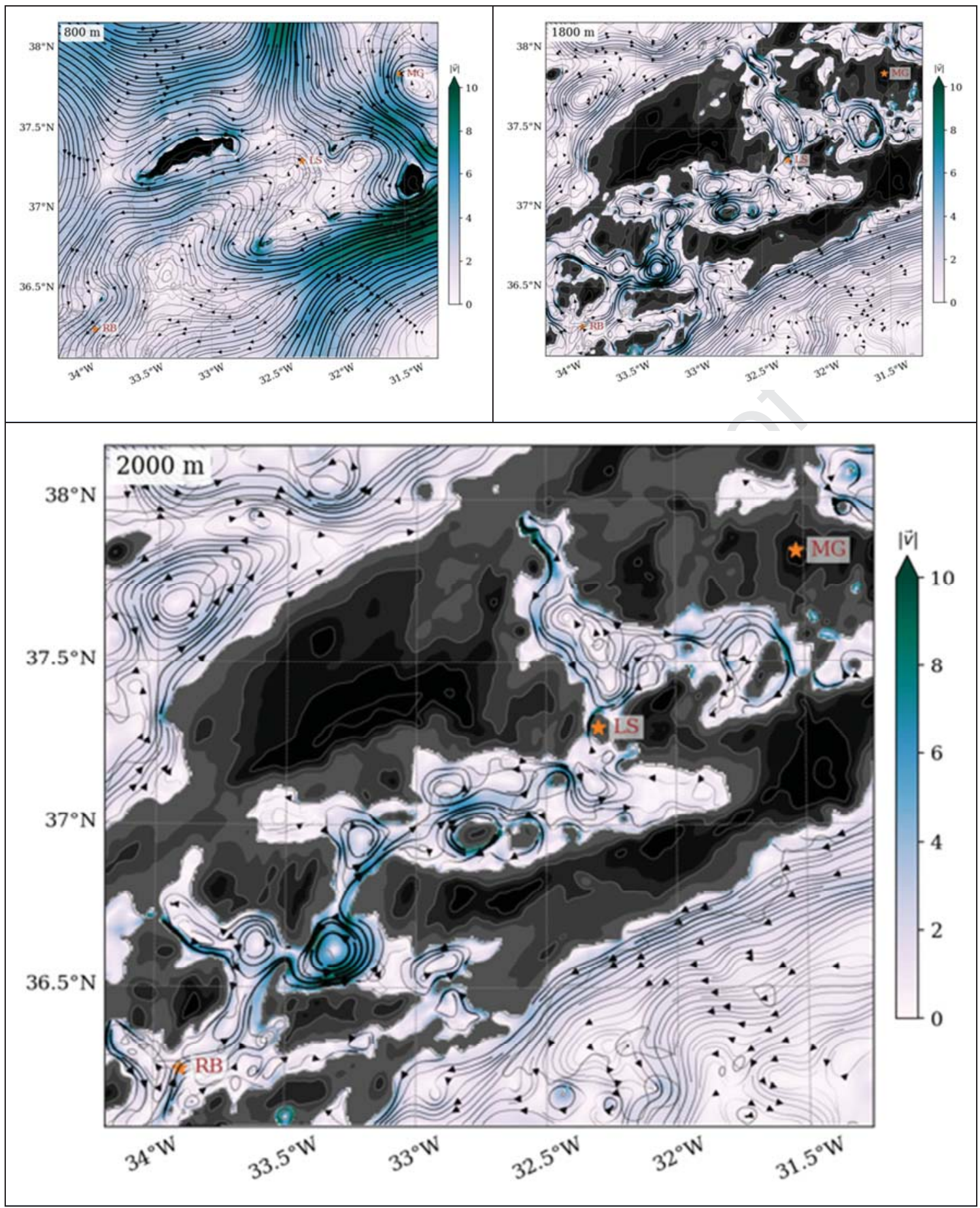

Figure 8. Currents over the northern mid-Atlantic Ridge (MAR) averaged over 4 months from a realistic numerical simulation at A. $800 \mathrm{~m}$, B. $1800 \mathrm{~m}$ and C. $2000 \mathrm{~m}$. Topography is indicated in dark gray when shallower than the depth of the plot and in light gray when deeper -at $500 \mathrm{~m}$ intervals. Currents are shown in black arrows (with the line width proportional to the amplitude) superimposed over the velocity magnitude in blue $(\mathrm{cm} / \mathrm{s}$, see the color bar on the right). Details on the numerical simulations are available in Vic et al. (2018). 


\section{Supplementary material}

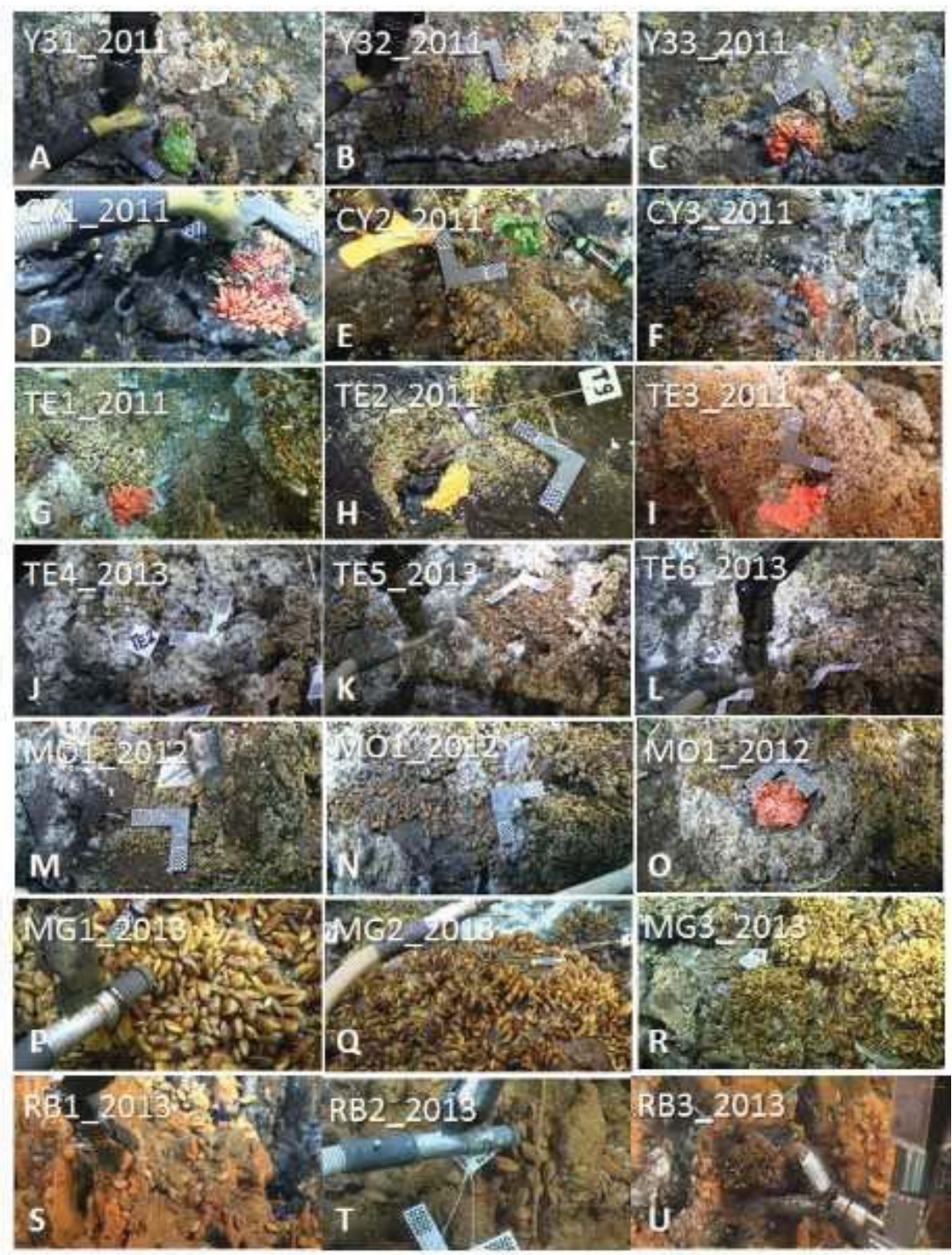

Figure S.1. Photos of the 21 sample locations from the three vent fields on the Mid-Atlantic Ridge. A-O : Lucky Strike, P-R : Menez Gwen and S-U : Rainbow. Samples were taken during several cruises on different research vessels using the ROV Victor6000. Targets, with visible $7 \times 7 \mathrm{~mm}$ checkerboards, were deployed to facilitate image calibration. For sampling, the Victor6000 ROV arm was used to sample the mussels after suction of small mobile fauna. A second suction sample was taken to clear the surface of the remaining fauna. 

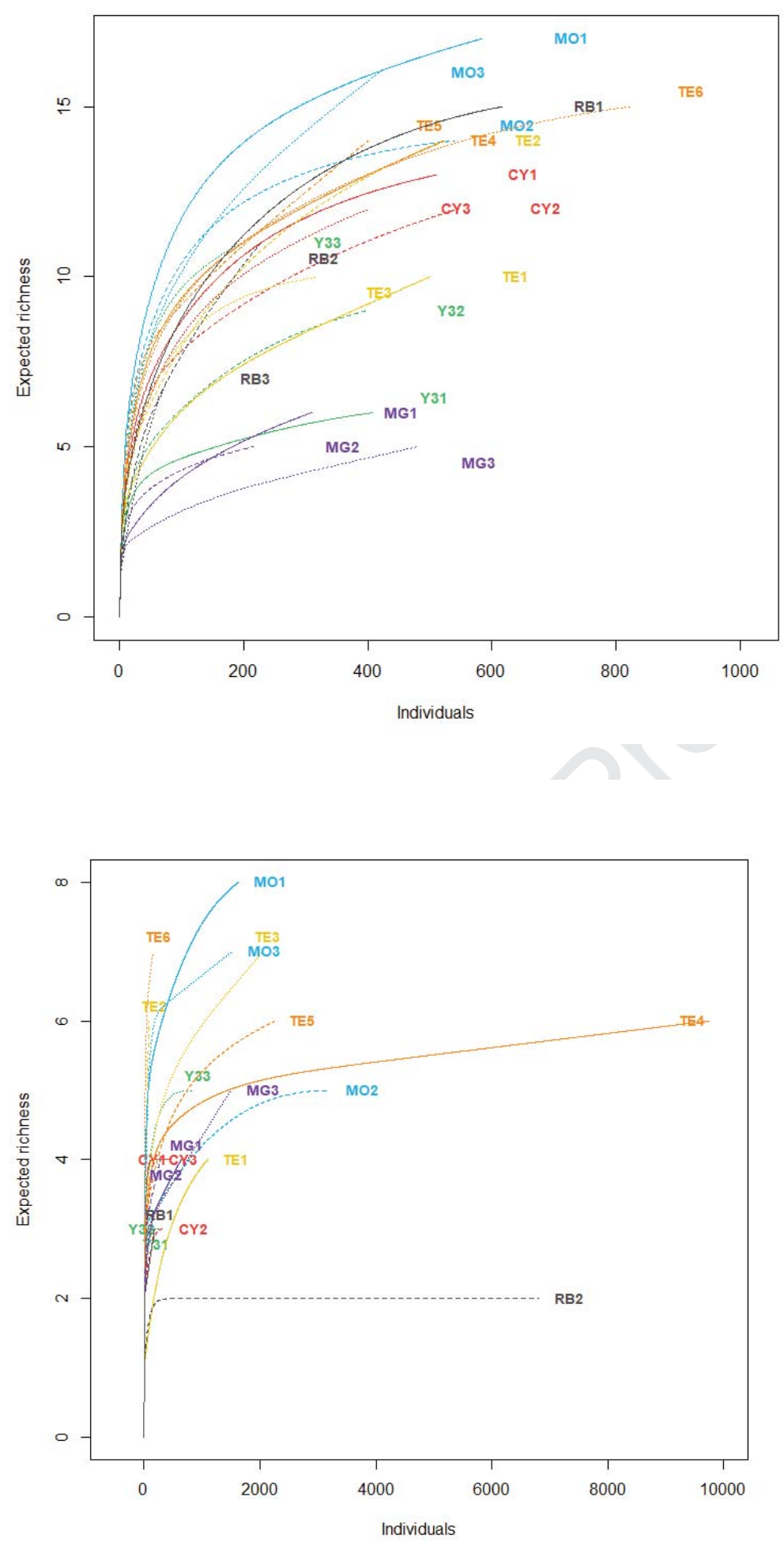

Figure S.2. Species accumulation curves of sampled macrofaunal taxa (A) and meiofaunal taxa (B). Color indicates the edifice: red: Cypress; green: Y3; yellow: Eiffel Tower 2011; blue: Montségur; orange: Eiffel Tower 2013; purple: Menez Gwen; black: Rainbow. Line type indicates the sample replicate: 1 : solid; 2 : dashed; 3 : dotted. 


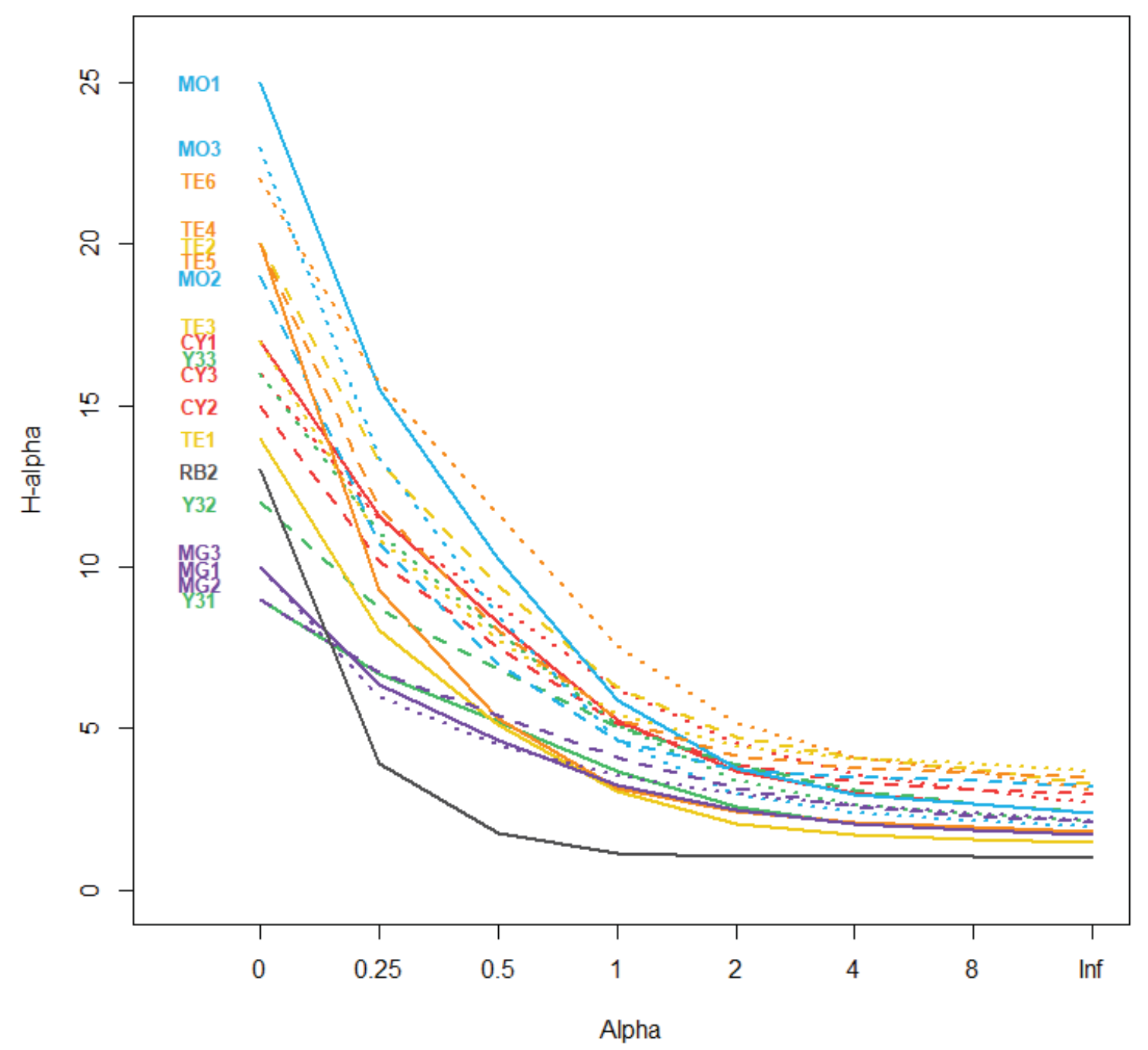

Figure S.3. Renyi's entropy profile $D^{\alpha}=\exp \left(H^{\alpha}\right)$. For $\alpha=0, D^{\alpha}=$ species richness. For $\alpha=1, D^{\alpha}=$ exponential of Shannon index. For $\alpha=2, D^{\alpha}=$ inverse of Simpson index. For $\alpha \rightarrow \operatorname{lnf}, D^{\alpha}=1 / P$, where $\mathrm{P}$ is the proportion of the dominant species. For $\alpha \rightarrow \operatorname{Inf}, \mathrm{D}^{\alpha}$ is an estimate of the evenness. Color indicates the edifice: red: Cypress; green: Y3; yellow: Eiffel Tower 2011; blue: Montségur; orange: Eiffel Tower 2013; purple: Menez Gwen; black: Rainbow. Line type indicates the sample replicate: 1: solid; 2: dashed; 3: dotted. 


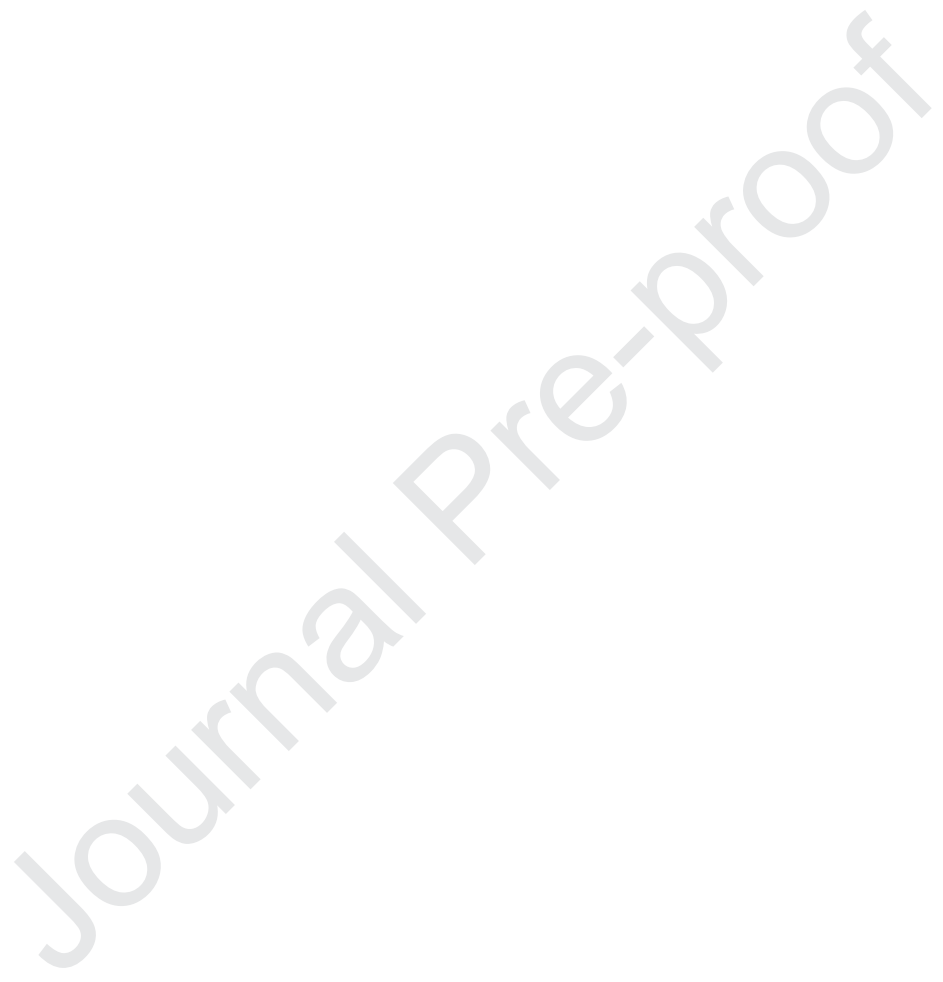




\section{Sarrazin et al. "Endogenous versus exogenous factors: what matters for vent} mussel communities?"

\section{Highlights}

- High consistency in species composition across different edifices at the field scale -Lucky Strike

- Various chemistry domains may influence local abiotic conditions and diversity patterns

- A "vent field signature" is observed across the three studied fields

- Distinct abundance and diversity patterns are observed, Menez Gwen being the most different

- Exogenous factors better explain the dissimilarities in faunal community structure

- Of particular interest are the current patterns between the three vent fields 


\section{Declaration of interests}

$\bigotimes$ The authors declare that they have no known competing financial interests or personal relationships that could have appeared to influence the work reported in this paper.

$\square$ The authors declare the following financial interests/personal relationships which may be considered as potential competing interests: 